Proceedings of the Edinburgh Mathematical Society (2006) 49, 173-199 (C)

DOI:10.1017/S0013091504000410 Printed in the United Kingdom

\title{
CYCLIC COHOMOLOGY OF PROJECTIVE LIMITS OF TOPOLOGICAL ALGEBRAS
}

\author{
ZINAIDA A. LYKOVA \\ School of Mathematics and Statistics, University of Newcastle, \\ Newcastle upon Tyne NE1 7RU, UK (z.a.lykova@newcastle.ac.uk)
}

(Received 4 June 2004)

\begin{abstract}
We present methods for the computation of the Hochschild and cyclic continuous cohomology and homology of some locally convex topological algebras. Let $\left(A_{\alpha}, T_{\alpha, \beta}\right)_{(\Lambda, \leqslant)}$ be a reduced projective system of complete Hausdorff locally convex algebras with jointly continuous multiplications, and let $A$ be the projective limit algebra $A=\lim _{\leftarrow} A_{\alpha}$. We prove that, for the continuous cyclic cohomology $H C^{*}$ and continuous periodic cohomology $H P^{*}$ of $A$ and $A_{\alpha}, \alpha \in \Lambda$, for all $n \geqslant 0, H C^{n}(A)=\lim _{\vec{\alpha}} H C^{n}\left(A_{\alpha}\right)$, the inductive limit of $H C^{n}\left(A_{\alpha}\right)$, and, for $k=0,1, H P^{k}(A)=\lim _{\vec{\alpha}} H P^{k}\left(A_{\alpha}\right)$. For a projective limit algebra $A=\lim _{\overleftarrow{m}} A_{m}$ of a countable reduced projective system $\left(A_{m}, T_{m, \ell}\right)_{\mathbb{N}}$ of Fréchet algebras, we also establish relations between the cyclic-type continuous homology of $A$ and $A_{m}, m \in \mathbb{N}$. For example, we show the exactness of the following short sequence for all $n \geqslant 0$ :

$$
0 \rightarrow \lim _{\check{m}}^{1} H C_{n+1}\left(A_{m}\right) \rightarrow H C_{n}(A) \rightarrow \lim _{\grave{m}} H C_{n}\left(A_{m}\right) \rightarrow 0
$$

We present a class of Fréchet algebras $A$ for which the continuous periodic cohomology $H P^{k}(A), k=0,1$, is isomorphic to the continuous cyclic cohomology $H C^{2 \ell+k}(A)$ starting from some integer $\ell$. We apply the above results to calculate the continuous cyclic-type homology and cohomology of some Fréchet locally $m$-convex algebras.
\end{abstract}

Keywords: continuous Hochschild and cyclic (co)homology; projective limits of topological locally convex algebras; Fréchet locally $m$-convex algebras

2000 Mathematics subject classification: Primary 19D55; 22E41; 46L80; $16 \mathrm{E} 40$

\section{Introduction}

In the spectral theory of commuting operators, in $K$-theory and in non-commutative differential geometry a prominent role is played by the continuous homology and cohomology groups of various topological algebras (see $[\mathbf{9}, \mathbf{1 4}]$ for references). There are a number of papers addressing the calculation of the cyclic-type continuous homology and cohomology groups of some Banach and topological algebras (see, for example, $[6,8-10,21,31,46,48])$. In the event that the topology of the algebra is given by a norm there is a well-developed and long-established homological theory $[\mathbf{2 0}, \mathbf{2 4}]$. However, it remains the case that the cyclic-type continuous homology and cohomology groups can only be computed for a restricted range of topological algebras. In this paper we present a tool for the computation of several types of continuous homology and cohomology groups 
of topological algebras. For Fréchet algebras, the basis of the method is to reduce the calculation of homology and cohomology groups of Fréchet algebras to the corresponding ones for known Banach algebras by a consistent use of projective limits. More generally, we consider a projective limit $A$ of a reduced projective system $\left(A_{\alpha}, T_{\alpha, \beta}\right)_{\alpha \in \Lambda}$ of complete Hausdorff locally convex algebras with jointly continuous multiplications, and reduce the calculation of its homology and cohomology groups to the corresponding ones for $A_{\alpha}$.

In $\S 2$ we present relations between Hochschild, cyclic and periodic cyclic homology and cohomology groups of mixed complexes in the category of locally convex spaces. In $\S 3$ we apply these relations to obtain results on various types of continuous Hochschild and cyclic homology and cohomology groups of locally convex and Fréchet algebras. In particular, we show that, for the class of Fréchet algebras $A$ with trivial continuous Hochschild cohomology groups $H H^{n}(A)$ for $n \geqslant N$, the continuous periodic cohomology groups $H P^{k}(A), k=0,1$, are isomorphic to the continuous cyclic cohomology groups $H C^{2 \ell+k}(A)$ starting from an integer $\ell \geqslant \frac{1}{2} N$. For example, this statement is true for such Fréchet algebras as $\mathcal{O}(\mathcal{U}), C^{\infty}(M)$ and $\mathcal{S}\left(\mathbb{R}^{m}\right)$ (see $\S 4.2$ ). Applications of the results from $\S 3$ and some well-known results from the homology of topological algebras to some classes of Fréchet and Banach algebras are given in $\S 4$. These provide new information on the continuous periodic cyclic homology and cohomology groups of those algebras.

One can use the Arens-Michael decomposition to reduce the calculation of the homology and cohomology groups of Fréchet algebras to the corresponding ones for Banach algebras. It is known that any Fréchet locally $m$-convex algebra $A$ is isomorphic to a projective limit of Banach algebras $A_{m}, m \in \mathbb{N}$, that is, $A=\lim _{\overleftarrow{m}} A_{m}$. The main purpose of this paper is to establish relations between the cyclic-type continuous cohomology and homology groups of $A$ and $A_{m}, m \in \mathbb{N}$, and to apply them to some natural classes of algebras. One of the difficulties of the topological case is that the quotient map from $A$ to $A_{m}$ is not surjective in general, but it has dense range. For example, for locally $C^{*}$-algebras those quotient maps are surjective [22]; the description of contractible locally $C^{*}$-algebras can be found in [16]. More generally, let $A$ be a projective limit of a reduced projective system $\left(A_{\alpha}, T_{\alpha, \beta}\right)_{\alpha \in \Lambda}$ of complete Hausdorff locally convex algebras with jointly continuous multiplications. In Theorem 5.1 we prove that for the cyclic continuous cohomology $H C^{*}$ and the periodic continuous cohomology $H P^{*}$ of $A$ and $A_{\alpha}, \alpha \in \Lambda$, up to isomorphism of linear spaces, for all $n \geqslant 0$,

$$
H C^{n}(A)=\lim _{\vec{\alpha}} H C^{n}\left(A_{\alpha}\right),
$$

the inductive limit of $H C^{n}\left(A_{\alpha}\right)$, and, for $k=0,1$,

$$
H P^{k}(A)=\lim _{\vec{\alpha}} H P^{k}\left(A_{\alpha}\right) .
$$

In Theorem 5.4, for a countable reduced projective system $\left(A_{m}, T_{m \ell}\right)_{m \in \mathbb{N}}$ of Fréchet algebras, we also establish relations between the cyclic-type continuous homology groups of $A=\lim _{\overleftarrow{m}} A_{m}$ and $A_{m}, m \in \mathbb{N}$. For example, we show that, for all $n \geqslant 0$, there exists a short exact sequence

$$
0 \rightarrow \lim _{\overleftarrow{m}}^{1} H C_{n+1}\left(A_{m}\right) \rightarrow H C_{n}(A) \rightarrow \lim _{\overleftarrow{m}} H C_{n}\left(A_{m}\right) \rightarrow 0
$$

where $\lim _{\grave{m}}^{1}$ is the first derived functor of the projective limit. 
There are similar short exact sequences for the following Hochschild homology groups $H_{*}^{\text {naive }}, H_{*}^{\text {bar }}, H H_{*}$, and for the periodic cyclic homology $H P_{*}$; see $\S 3$ for the definitions of these homology groups. In $\S 6$ we apply the results of $\S \S 3-5$ to Fréchet locally $m$ convex algebras. For example, applications show that, for the Fréchet locally $C^{*}$-algebra $\mathcal{L}(H)$ of continuous linear operators on an infinite-dimensional locally Hilbert space $H$, the continuous cyclic and periodic cyclic homology and cohomology groups of $\mathcal{L}(H)$ are trivial. We also show that, for the Fréchet algebra $C(\mathbb{R})$ of continuous complex-valued functions on $\mathbb{R}$, the continuous cyclic and periodic cyclic homology and cohomology groups of $C(\mathbb{R})$ are the following:

$$
\begin{gathered}
H P_{0}(C(\mathbb{R}))=H C_{2 \ell}(C(\mathbb{R}))=C(\mathbb{R}), \quad H P_{1}(C(\mathbb{R}))=H C_{2 \ell+1}(C(\mathbb{R}))=\{0\}, \\
H P^{0}(C(\mathbb{R}))=H C^{2 \ell}(C(\mathbb{R}))=\lim _{\vec{k}} M([-k, k])=M_{\mathrm{c}}(\mathbb{R})
\end{gathered}
$$

the complex linear space of $\mathbb{C}$-valued Radon measures on $\mathbb{R}$ with compact supports, and

$$
H P^{1}(C(\mathbb{R}))=H C^{2 \ell+1}(C(\mathbb{R}))=\{0\} \quad \text { for all } \ell \geqslant 0 .
$$

\section{Homologies of mixed complexes in the category of locally convex spaces}

The category of locally convex spaces and continuous linear operators is denoted by $\mathcal{L C S}$. A Fréchet space is a metrizable and complete locally convex topological vector space over $\mathbb{C}$ or $\mathbb{R}$. The category of Fréchet spaces and continuous linear operators is denoted by ' $\mathcal{F} r$ '. Throughout the paper 'id' denotes the identity operator.

There is a powerful method based on mixed complexes for the study of the cyclic-type homology groups (see papers by Kassel [25], Cuntz and Quillen [11] and Cuntz [10]). We shall present this method for the category $\mathcal{L C S}$ of locally convex spaces and continuous linear operators; see [5] for the category of Fréchet spaces. A mixed complex $(\mathcal{M}, b, B)$ in the category $\mathcal{L C S}$ is a family $\mathcal{M}=\left\{M_{n}\right\}_{n \geqslant 0}$ of locally convex spaces $M_{n}$ equipped with continuous linear operators $b_{n}: M_{n} \rightarrow M_{n-1}$ and $B_{n}: M_{n} \rightarrow M_{n+1}$, which satisfy the identities $b^{2}=b B+B b=B^{2}=0$. We assume that in degree zero the differential $b$ is identically equal to zero. We arrange the mixed complex $(\mathcal{M}, b, B)$ in the double complex

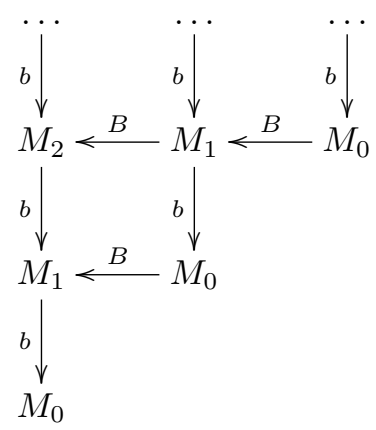

There are three types of homology theory that can be naturally associated with a mixed complex. The Hochschild homology $H_{*}^{b}(\mathcal{M})$ of $(\mathcal{M}, b, B)$ is the homology of the chain 
complex $(\mathcal{M}, b)$, that is,

$$
H_{n}^{b}(\mathcal{M})=H_{n}(\mathcal{M}, b)=\operatorname{Ker}\left\{b_{n}: M_{n} \rightarrow M_{n-1}\right\} / \operatorname{Im}\left\{b_{n+1}: M_{n+1} \rightarrow M_{n}\right\} .
$$

To define the cyclic homology of $(\mathcal{M}, b, B)$, let us denote by $\mathcal{B}_{\mathrm{c}} \mathcal{M}$ the total complex of the above double complex, that is,

$$
\cdots \longrightarrow\left(\mathcal{B}_{\mathrm{c}} \mathcal{M}\right)_{n} \stackrel{b+B}{\longrightarrow}\left(\mathcal{B}_{\mathrm{c}} \mathcal{M}\right)_{n-1} \longrightarrow \cdots \stackrel{b+B}{\longrightarrow}\left(\mathcal{B}_{\mathrm{c}} \mathcal{M}\right)_{0} \longrightarrow 0,
$$

where the spaces

$$
\left(\mathcal{B}_{\mathrm{c}} \mathcal{M}\right)_{0}=M_{0}, \ldots,\left(\mathcal{B}_{\mathrm{c}} \mathcal{M}\right)_{2 k-1}=M_{1} \oplus M_{3} \oplus \cdots \oplus M_{2 k-1}
$$

and

$$
\left(\mathcal{B}_{\mathrm{c}} \mathcal{M}\right)_{2 k}=M_{0} \oplus M_{2} \oplus \cdots \oplus M_{2 k}
$$

are equipped with the product topology, and the continuous linear operators $b+B$ are defined by

$$
(b+B)\left(y_{0}, \ldots, y_{2 k}\right)=\left(b y_{2}+B y_{0}, \ldots, b y_{2 k}+B y_{2 k-2}\right)
$$

and

$$
(b+B)\left(y_{1}, \ldots, y_{2 k+1}\right)=\left(b y_{1}, \ldots, b y_{2 k+1}+B y_{2 k-1}\right) .
$$

The cyclic homology of $(\mathcal{M}, b, B)$ is defined to be $H_{*}\left(\mathcal{B}_{\mathrm{c}} \mathcal{M}, b+B\right)$. It is denoted by $H_{*}^{\mathrm{c}}(\mathcal{M}, b, B)$.

The periodic cyclic homology of $(\mathcal{M}, b, B)$ is defined in terms of the complex

$$
\cdots \longrightarrow\left(\mathcal{B}_{\mathrm{p}} \mathcal{M}\right)_{\mathrm{ev}} \stackrel{b+B}{\longrightarrow}\left(\mathcal{B}_{\mathrm{p}} \mathcal{M}\right)_{\text {odd }} \stackrel{b+B}{\longrightarrow}\left(\mathcal{B}_{\mathrm{p}} \mathcal{M}\right)_{\mathrm{ev}} \stackrel{b+B}{\longrightarrow}\left(\mathcal{B}_{\mathrm{p}} \mathcal{M}\right)_{\text {odd }} \longrightarrow \cdots
$$

where even/odd chains are elements of the product spaces

$$
\left(\mathcal{B}_{\mathrm{p}} \mathcal{M}\right)_{\mathrm{ev}}=\prod_{n \geqslant 0} M_{2 n} \quad \text { and } \quad\left(\mathcal{B}_{\mathrm{p}} \mathcal{M}\right)_{\text {odd }}=\prod_{n \geqslant 0} M_{2 n+1},
$$

respectively. The spaces $\left(\mathcal{B}_{\mathrm{p}} \mathcal{M}\right)_{\text {ev/odd }}$ are locally convex spaces with respect to the product topology $[\mathbf{2 7}, \S 18.3 .(5)]$. The continuous differential $b+B$ is defined as an obvious extension of the above. The periodic cyclic homology of $(\mathcal{M}, b, B)$ is $H_{\nu}^{\mathrm{p}}(\mathcal{M}, b, B)=$ $H_{\nu}\left(\mathcal{B}_{\mathrm{p}} \mathcal{M}, b+B\right)$, where $\nu \in \mathbb{Z} / 2 \mathbb{Z}$.

There are also three types of cyclic cohomology theory associated with the mixed complex, obtained when one replaces the chain complex of locally convex spaces by its dual complex of strong dual spaces. For example, the cyclic cohomology associated with the mixed complex $(\mathcal{M}, b, B)$ is defined to be the cohomology of the dual complex $\left(\left(\mathcal{B}_{\mathrm{c}} \mathcal{M}\right)^{\prime}, b^{*}+B^{*}\right)$ of strong dual spaces and dual operators; it is denoted by $H_{\mathrm{c}}^{*}\left(\mathcal{M}^{\prime}, b^{*}, B^{*}\right)$. 
One of the important homological properties of mixed complexes is the existence of the Connes-Tsygan long exact sequence associated with $(\mathcal{M}, b, B)$. First we define the Connes periodicity operator

$$
S:\left(\mathcal{B}_{\mathrm{c}} \mathcal{M}\right)_{n} \rightarrow\left(\mathcal{B}_{\mathrm{c}} \mathcal{M}\right)_{n-2}
$$

by the formulae

$$
S\left(y_{0}, \ldots, y_{2 n-2}, y_{2 n}\right)=\left(y_{0}, \ldots, y_{2 n-2}\right) \quad \text { and } \quad S\left(y_{1}, \ldots, y_{2 n+1}\right)=\left(y_{1}, \ldots, y_{2 n-1}\right)
$$

for $n \geqslant 1$. We put $S M_{0}=\{0\}, S M_{1}=\{0\}$. It is clear that $S$ is continuous in the product topology. We then have the following short exact sequence of complexes of locally convex spaces:

$$
0 \rightarrow(\mathcal{M}, b) \stackrel{I}{\rightarrow}\left(\mathcal{B}_{\mathrm{c}} \mathcal{M}, b+B\right) \stackrel{S}{\rightarrow}\left(\mathcal{B}_{\mathrm{c}} \mathcal{M}[2],(b+B)[2]\right) \rightarrow 0,
$$

where $I$ is the natural inclusion. Throughout this paper, for a chain complex $(K, d)$, the complex $(K[m], d[m])$ is defined by $(K[m])_{q}=K_{q-m}$ and $(d[m])_{q}=(-1)^{m} d_{q-m}$. Hence, there is a long exact sequence

$$
\cdots \rightarrow H_{n+2}^{b}(\mathcal{M}) \rightarrow H_{n+2}^{\mathrm{c}}(\mathcal{M}, b, B) \rightarrow H_{n}^{\mathrm{c}}(\mathcal{M}, b, B) \rightarrow H_{n+1}^{b}(\mathcal{M}) \rightarrow \cdots
$$

of homology groups. Recall that in the category of Fréchet spaces induced maps of the sequence (2.3) are continuous [20, Theorem 0.5.7].

It is easy to see that the short sequence of dual complexes:

$$
0 \leftarrow\left(\mathcal{M}^{\prime}, b^{*}\right) \stackrel{I^{\prime}}{\longleftarrow}\left(\left(\mathcal{B}_{\mathrm{c}} \mathcal{M}\right)^{\prime}, b^{*}+B^{*}\right) \stackrel{S^{\prime}}{\longleftarrow}\left(\left(\mathcal{B}_{\mathrm{c}} \mathcal{M}[2]\right)^{\prime},\left(b^{*}+B^{*}\right)[2]\right) \leftarrow 0
$$

is exact too. Hence, there is a long exact sequence of cohomology groups

$$
\cdots \rightarrow H_{b}^{n}\left(\mathcal{M}^{\prime}\right) \rightarrow H_{\mathrm{c}}^{n-1}\left(\mathcal{M}^{\prime}, b^{*}, B^{*}\right) \rightarrow H_{\mathrm{c}}^{n+1}\left(\mathcal{M}^{\prime}, b^{*}, B^{*}\right) \rightarrow H_{b}^{n+1}\left(\mathcal{M}^{\prime}\right) \rightarrow \cdots .
$$

Proposition 2.1. Let $(\mathcal{M}, b, B)$ be a mixed complex of locally convex spaces. Then, for any even integer $N$, say $N=2 K$, and the following assertions, we have (i) $)_{N} \Rightarrow$ $(\text { ii })_{N} \Rightarrow(\text { i })_{N+1}$ and $(\text { i })_{N} \Rightarrow(\text { iii })_{N}$ :

(i) $)_{N}$ for all $n \geqslant N, H_{n}^{b}(\mathcal{M})=\{0\}$;

$(\text { ii })_{N}$ for all $k \geqslant K$, up to isomorphism of linear spaces,

$$
H_{2 k}^{\mathrm{c}}(\mathcal{M}, b, B)=H_{N}^{\mathrm{c}}(\mathcal{M}, b, B)
$$

and

$$
H_{2 k+1}^{\mathrm{c}}(\mathcal{M}, b, B)=H_{N-1}^{\mathrm{c}}(\mathcal{M}, b, B)
$$

$(\text { iii })_{N}$ up to isomorphism of linear spaces,

$$
H_{0}^{\mathrm{p}}(\mathcal{M}, b, B)=H_{N}^{\mathrm{c}}(\mathcal{M}, b, B)
$$

and

$$
H_{1}^{\mathrm{p}}(\mathcal{M}, b, B)=H_{N-1}^{\mathrm{c}}(\mathcal{M}, b, B)
$$


Proof. (i) $)_{N} \Rightarrow(\mathrm{ii})_{N}$ and $(\mathrm{ii})_{N} \Rightarrow(\mathrm{i})_{N+1}$ follow from the exactness of the ConnesTsygan long exact sequence for the homology groups (2.3) and [30, Lemma 3.1].

To prove $(\mathrm{i})_{N} \Rightarrow(\text { iii })_{N}$ we shall use methods from [6, Theorem 3] and [5, Lemma 1]. One can see that there is a short exact sequence of complexes:

$$
0 \rightarrow\left(\mathcal{B}_{\mathrm{p}, \mathrm{c}} \mathcal{M}, b+B\right) \rightarrow\left(\mathcal{B}_{\mathrm{p}} \mathcal{M}, b+B\right) \stackrel{F}{\rightarrow}\left(\mathcal{B}_{\mathrm{c}} \mathcal{M}, b+B\right) \rightarrow 0
$$

where $\left(\mathcal{B}_{\mathrm{p}, \mathrm{c}} \mathcal{M}, b+B\right)$ is by definition the sub-complex Ker $F$ of the complex $\left(\mathcal{B}_{\mathrm{p}} \mathcal{M}, b+B\right)$ and $F$ is defined by

$$
F_{2 n}:\left(\mathcal{B}_{\mathrm{p}} \mathcal{M}\right)_{\mathrm{ev}} \rightarrow\left(\mathcal{B}_{\mathrm{c}} \mathcal{M}\right)_{2 n}:\left(y_{0}, \ldots, y_{2 k}, \ldots\right) \mapsto\left(y_{0}, \ldots, y_{2 n}\right),
$$

and

$$
F_{2 n+1}:\left(\mathcal{B}_{\mathrm{p}} \mathcal{M}\right)_{\mathrm{odd}} \rightarrow\left(\mathcal{B}_{\mathrm{c}} \mathcal{M}\right)_{2 n+1}:\left(y_{1}, \ldots, y_{2 k+1}, \ldots\right) \mapsto\left(y_{1}, \ldots, y_{2 n+1}\right)
$$

Hence, there is a long exact sequence of homology groups

$$
\begin{aligned}
\cdots \longrightarrow H_{n}\left(\mathcal{B}_{\mathrm{p}, \mathrm{c}} \mathcal{M}, b+B\right) \longrightarrow H_{n}^{\mathrm{p}}(\mathcal{M}, b, B) \stackrel{H(F)_{n}}{\longrightarrow} & H_{n}^{\mathrm{c}}(\mathcal{M}, b, B) \longrightarrow \\
& H_{n-1}\left(\mathcal{B}_{\mathrm{p}, \mathrm{c}} \mathcal{M}, b+B\right) \longrightarrow \cdots
\end{aligned}
$$

We claim that, for all $n \geqslant N, H(F)_{n}$ is an isomorphism. Therefore, by [30, Lemma 3.12], it follows from the exactness of the sequence (2.7) that, for all $n \geqslant N$, $H_{n}\left(\mathcal{B}_{\mathrm{p}, \mathrm{c}} \mathcal{M}, b+B\right)=\{0\}$.

To show that $H(F)_{n}$ is surjective, assume that $n=2 k$ where $k \geqslant K$ and let $\left(m_{0}, \ldots, m_{n}\right) \in\left(\mathcal{B}_{\mathrm{c}} \mathcal{M}\right)_{n}$ be a cycle, that is, $\left(b m_{2}+B m_{0}, \ldots, b m_{2 k}+B m_{2 k-2}\right)=0$ and, in particular, $b m_{2 k}=-B m_{2 k-2}$. Further, since $b B=-B b$ and $B B=0$, we can see that

$$
b B m_{2 k}=-B b m_{2 k}=B^{2} m_{2 k-2}=0 .
$$

Hence $-B m_{2 k} \in\left(\mathcal{M}_{2 k+1}, b\right)$ is a cycle. By assumption, for all $n \geqslant 2 K$, the homology of $(\mathcal{M}, b)$ vanishes, and so there exists $y_{2 k+2} \in M_{2 k+2}$ such that $b y_{2 k+2}=-B m_{2 k}$. By the inductive hypothesis, there exists $y_{2 k+2 \ell} \in M_{2 k+2 \ell}$ such that $b y_{2 k+2 \ell}=-B y_{2 k+2(\ell-1)}$. Again, since $b B=-B b$ and $B B=0$, we can see that

$$
b B y_{2 k+2 \ell}=-B b y_{2 k+2 \ell}=B^{2} y_{2 k+2(\ell-1)}=0 .
$$

Hence, $-B y_{2 k+2 \ell} \in\left(\mathcal{M}_{2 k+2 \ell+1}, b\right)$ is a cycle. By assumption, for all $n \geqslant 2 K$, the homology of $(\mathcal{M}, b)$ vanishes, and so there exists $y_{2 k+2(\ell+1)} \in M_{2 k+2(\ell+1)}$ such that $b y_{2 k+2(\ell+1)}=-B y_{2 k+2 \ell}$. Hence we can construct a chain

$$
\left(m_{0}, \ldots, m_{2 k}, y_{2 k+2}, y_{2 k+4}, \ldots\right) \in(\mathcal{B M})_{\mathrm{ev}}^{\mathrm{p}}
$$

such that

$$
(b+B)\left(m_{0}, \ldots, m_{2 k}, y_{2 k+2}, y_{2 k+4}, \ldots\right)=0
$$


and

$$
H(F)_{2 k}\left(m_{0}, \ldots, m_{2 k}, y_{2 k+2}, y_{2 k+4}, \ldots\right)=\left(m_{0}, \ldots, m_{2 k}\right)+\operatorname{Im}(b+B) .
$$

To show that $H(F)_{n}$ is injective, assume that $n=2 k$ where $k \geqslant K$ and let

$$
\left(y_{0}, \ldots, y_{2 \ell}, \ldots\right) \in\left(\mathcal{B}_{\mathrm{p}} \mathcal{M}\right)_{\mathrm{ev}}
$$

be a cycle, that is,

$$
\left(b y_{2}+B y_{0}, \ldots, b y_{2 k}+B y_{2 k-2}, \ldots\right)=0 .
$$

In particular, $b y_{2 k+2}+B y_{2 k}=0$ and

$$
H(F)_{n}\left(y_{0}, \ldots, y_{2 \ell}, \ldots\right)=\left(y_{0}, \ldots, y_{2 k}\right)+\operatorname{Im}(b+B)=0,
$$

that is, there is a chain $\left(v_{1}, \ldots, v_{2 k+1}\right) \in\left(\mathcal{B}_{\mathrm{c}} \mathcal{M}\right)_{2 k+1}$ such that

$$
(b+B)\left(v_{1}, \ldots, v_{2 k+1}\right)=\left(b v_{1}, b v_{3}+B v_{1}, \ldots, b v_{2 k+1}+B v_{2 k-1}\right)=\left(y_{0}, \ldots, y_{2 k}\right) .
$$

Further, since $b B=-B b$ and $B B=0$, it follows that

$$
\begin{aligned}
b\left(y_{2 k+2}-B v_{2 k+1}\right) & =b y_{2 k+2}+B b v_{2 k+1} \\
& =b y_{2 k+2}+B\left(y_{2 k}-B v_{2 k-1}\right) \\
& =-B y_{2 k}+B y_{2 k}-B^{2} v_{2 k-1} \\
& =0 .
\end{aligned}
$$

Hence $y_{2 k+2}-B v_{2 k+1} \in\left(\mathcal{M}_{2 k+2}, b\right)$ is a cycle. By assumption, for all $n \geqslant 2 K$, the homology of $(\mathcal{M}, b)$ vanishes, and so there exists $v_{2 k+3} \in M_{2 k+3}$ such that $b v_{2 k+3}=$ $y_{2 k+2}-B v_{2 k+1}$, that is, $y_{2 k+2}=b v_{2 k+3}+B v_{2 k+1}$. By the inductive hypothesis, there exists a chain $\left(v_{1}, \ldots, v_{2 k+1}, v_{2 k+3}, \ldots\right) \in\left(\mathcal{B}_{\mathrm{p}} \mathcal{M}\right)_{\text {odd }}$ such that

$$
\begin{aligned}
(b+B)\left(v_{1}, \ldots, v_{2 k+1}, v_{2 k+3}, \ldots\right) & =\left(b v_{1}, b v_{3}+B v_{1}, \ldots, b v_{2 k+1}+B v_{2 k-1}, \ldots\right) \\
& =\left(y_{0}, \ldots, y_{2 k}, y_{2 k+1}, \ldots\right) .
\end{aligned}
$$

Thus, $H(F)_{n}$ is injective.

Similar arguments work in the odd case.

Remark 2.2. If $(\mathcal{M}, b, B)$ is a mixed complex of Fréchet spaces, by the previous proposition and by [20, Lemma 0.5.9] or [2, Lemma 7.1.32], $H(F)_{n}$ is a topological isomorphism.

The proof of the following statement for dual mixed complexes of locally convex spaces is similar to that for homology groups of mixed complexes (Proposition 2.1).

Proposition 2.3. Let $(\mathcal{M}, b, B)$ be a mixed complex of locally convex spaces and let $\left(\mathcal{M}^{\prime}, b^{*}, B^{*}\right)$ be the dual complex of strong dual spaces. Then, for any even integer $N$, say $N=2 K$, and the following assertions, we have $(\mathrm{i})_{N} \Rightarrow(\mathrm{ii})_{N} \Rightarrow(\mathrm{i})_{N+1}$ and $(\mathrm{i})_{N} \Rightarrow(\mathrm{iii})_{\mathrm{N}}$ :

(i) $N$ for all $n \geqslant N$,

$$
H_{b}^{n}\left(\mathcal{M}^{\prime}, b^{*}\right)=\{0\}
$$


$(\text { ii })_{N}$ for all $k \geqslant K$, up to isomorphism of linear spaces,

$$
H_{\mathrm{c}}^{2 k}\left(\mathcal{M}^{\prime}, b^{*}, B^{*}\right)=H_{\mathrm{c}}^{N}\left(\mathcal{M}^{\prime}, b^{*}, B^{*}\right)
$$

and

$$
H_{\mathrm{c}}^{2 k+1}\left(\mathcal{M}^{\prime}, b^{*}, B^{*}\right)=H_{\mathrm{c}}^{N-1}\left(\mathcal{M}^{\prime}, b^{*}, B^{*}\right)
$$

$(\text { iii) })_{N}$ up to isomorphism of linear spaces,

$$
H_{\mathrm{p}}^{0}\left(\mathcal{M}^{\prime}, b^{*}, B^{*}\right)=H_{\mathrm{c}}^{N}\left(\mathcal{M}^{\prime}, b^{*}, B^{*}\right)
$$

and

$$
H_{\mathrm{p}}^{1}\left(\mathcal{M}^{\prime}, b^{*}, B^{*}\right)=H_{\mathrm{c}}^{N-1}\left(\mathcal{M}^{\prime}, b^{*}, B^{*}\right) .
$$

Further we shall need the following technical result for Fréchet spaces, which extends a result of Johnson for the Banach case [24, Corollary 1.3]; one can also find it in [20, Proposition II.5.29].

Proposition 2.4. Let $(\mathcal{M}, b)$ be a complex of Fréchet spaces and let $N \in \mathbb{N}$. Then the following statements are equivalent:

(i) $H_{n}(\mathcal{M}, b)=\{0\}$ for all $n \geqslant N$ and $H_{N-1}(\mathcal{M})$ is Hausdorff;

(ii) $H^{n}\left(\mathcal{M}^{\prime}, b^{*}\right)=\{0\}$ for all $n \geqslant N$.

The proof depends on the open mapping principle and the Hahn-Banach theorem.

\section{Relations between cyclic-type homology groups for locally convex algebras}

Let $A$ be a complete locally convex algebra, not necessarily unital. By a locally convex algebra we shall mean an algebra $A$, which is a locally convex topological vector space in such a way that the ring multiplication in $A$ is jointly continuous. One can consult the books by Loday [29] or Connes [9] on cyclic-type homological theory.

The continuous bar and 'naive' Hochschild homology of $A$ are defined respectively as

$$
H_{*}^{\text {bar }}(A)=H_{*}\left(\mathcal{C}(A), b^{\prime}\right) \quad \text { and } \quad H_{*}^{\text {naive }}(A)=H_{*}(\mathcal{C}(A), b),
$$

where $\mathcal{C}_{n}(A)=A^{\hat{\otimes}(n+1)}, \hat{\otimes}$ is the completed projective tensor product, and the differentials $b, b^{\prime}$ are given by

$$
b^{\prime}\left(a_{0} \otimes \cdots \otimes a_{n}\right)=\sum_{i=0}^{n-1}(-1)^{i}\left(a_{0} \otimes \cdots \otimes a_{i} a_{i+1} \otimes \cdots \otimes a_{n}\right)
$$

and

$$
b\left(a_{0} \otimes \cdots \otimes a_{n}\right)=b^{\prime}\left(a_{0} \otimes \cdots \otimes a_{n}\right)+(-1)^{n}\left(a_{n} a_{0} \otimes \cdots \otimes a_{n-1}\right) .
$$


Note that $H_{*}^{\text {naive }}(A)$ is just another way of writing $\mathcal{H}_{*}(A, A)$, the continuous homology of $A$ with coefficients in $A$, as described in $[\mathbf{2 0}, \mathbf{2 4}]$.

We denote by $A_{+}$the unitization of a complete locally convex algebra $A$ and consider the mixed complex $\left(\bar{\Omega} A_{+}, \tilde{b}, \tilde{B}\right)$ in $\mathcal{F} r$, where $\bar{\Omega}^{n} A_{+}=A^{\hat{\otimes}(n+1)} \oplus A^{\hat{\otimes} n}$ and

$$
\tilde{b}=\left(\begin{array}{cc}
b & 1-\lambda \\
0 & -b^{\prime}
\end{array}\right) ; \quad \tilde{B}=\left(\begin{array}{cc}
0 & 0 \\
N & 0
\end{array}\right),
$$

where $\lambda\left(a_{1} \otimes \cdots \otimes a_{n}\right)=(-1)^{n-1}\left(a_{n} \otimes a_{1} \otimes \cdots \otimes a_{n-1}\right)$ and $N=\mathrm{id}+\lambda+\cdots+\lambda^{n-1}[\mathbf{2 9}$, 1.4.5]. The continuous Hochschild homology of $A$, the continuous cyclic homology of $A$ and the continuous periodic cyclic homology of $A$ are defined by

$$
H H_{*}(A)=H_{*}^{b}\left(\bar{\Omega} A_{+}, \tilde{b}, \tilde{B}\right), \quad H C_{*}(A)=H_{*}^{\mathrm{c}}\left(\bar{\Omega} A_{+}, \tilde{b}, \tilde{B}\right)
$$

and

$$
H P_{*}(A)=H_{*}^{\mathrm{p}}\left(\bar{\Omega} A_{+}, \tilde{b}, \tilde{B}\right) .
$$

There is also a cyclic cohomology theory associated with a complete locally convex algebra $A$, obtained when one replaces the chain complexes of $A$ by their dual complexes of strong dual spaces. For example, the continuous bar cohomology $H_{\mathrm{bar}}^{n}(A)$ of $A$ is the cohomology of the dual complex $\left(\mathcal{C}(A)^{\prime},\left(b^{\prime}\right)^{*}\right)$ of $\left(\mathcal{C}(A), b^{\prime}\right)[\mathbf{9}, \mathbf{2 9}]$.

By extending results of $[\mathbf{2 4}]$ one can prove that, for a Fréchet algebra $A$ with a leftor right-bounded approximate identity, $H_{\text {bar }}^{n}(A)=\{0\}$ for all $n \geqslant 0$, and so, by $[\mathbf{5}$, Lemma 2], $H_{n}^{\text {bar }}(A)=\{0\}$ for all $n \geqslant 0$.

For example, $H_{\mathrm{bar}}^{n}(A)=\{0\}$ for all $n \geqslant 0$, for any $C^{*}$-algebra $A$, because $C^{*}$-algebras have bounded approximate identities. It is also true for the Banach algebra $\mathcal{K}(E)$ of compact operators on a Banach space $E$ with the bounded compact approximation property. This algebra $\mathcal{K}(E)$ has a left-bounded approximate identity [13]. For more examples of Banach algebras with a left- or right-bounded approximate identity (see, for example, $[\mathbf{3 8}, \S 5.1])$.

We note also the following short exact sequence of complexes of complete locally convex spaces and continuous linear operators

$$
0 \rightarrow(\mathcal{C}(A), b) \rightarrow\left(\bar{\Omega} A_{+}, \tilde{b}\right) \rightarrow\left(\mathcal{C}(A)[1], b^{\prime}[1]\right) \rightarrow 0,
$$

which leads to a long exact homology sequence connecting the three homology groups

$$
\cdots \rightarrow H_{n}^{\text {bar }}(A) \rightarrow H_{n}^{\text {naive }}(A) \rightarrow H H_{n}(A) \rightarrow H_{n-1}^{\text {bar }}(A) \rightarrow \cdots .
$$

This shows that $H_{n}^{\text {naive }}(A)=H H_{n}(A)$ for all $n \geqslant 0$ if and only if $H_{n}^{\text {bar }}(A)=\{0\}$ for all $n \geqslant 0$. Recall that in the category of Fréchet spaces induced maps of the sequence (3.2) are continuous [20, Theorem 0.5.7].

It is easy to see that the short sequence of dual complexes

$$
0 \leftarrow\left(\mathcal{C}(A)^{\prime}, b^{*}\right) \leftarrow\left(\left(\bar{\Omega} A_{+}\right)^{\prime}, \tilde{b}^{*}\right) \leftarrow\left((\mathcal{C}(A)[1])^{\prime},\left(b^{\prime}[1]\right)^{*}\right) \leftarrow 0
$$


is exact too. Hence, there is a long exact cohomology sequence connecting the three cohomology groups

$$
\cdots \rightarrow H_{\text {bar }}^{n-1}(A) \rightarrow H H^{n}(A) \rightarrow H_{\text {naive }}^{n}(A) \rightarrow H_{\text {bar }}^{n}(A) \rightarrow \cdots .
$$

This shows that $H_{\text {naive }}^{n}(A)=H H^{n}(A)$ for all $n \geqslant 0$ if and only if $H_{\text {bar }}^{n}(A)=\{0\}$ for all $n \geqslant 0$.

In view of the existence of the long exact sequences (2.3) and (2.5), there are long exact Connes sequences for complete locally convex algebras:

$$
\cdots \rightarrow H H_{n}(A) \rightarrow H C_{n}(A) \rightarrow H C_{n-2}(A) \rightarrow H H_{n-1}(A) \rightarrow \cdots
$$

and

$$
\cdots \rightarrow H H^{n}(A) \rightarrow H C^{n-1}(A) \rightarrow H C^{n+1}(A) \rightarrow H H^{n+1}(A) \rightarrow \cdots .
$$

Therefore, by virtue of Proposition 2.4, the five lemmas and the above results, we can prove the following theorem, which is a generalization of [5, Theorem 1].

Theorem 3.1. Let $A$ be a complete locally convex algebra. Then, for the following statements, (i) is equivalent to (ii); (iii) is equivalent to (iv); and, for Fréchet algebras $A$, (ii) is equivalent to (iii).

(i) There exists a long exact Connes-Tsygan sequence of continuous homology groups

$$
\cdots \rightarrow H_{n}^{\text {naive }}(A) \rightarrow H C_{n}(A) \rightarrow H C_{n-2}(A) \rightarrow H_{n-1}^{\text {naive }}(A) \rightarrow \cdots ;
$$

(ii) $H_{n}^{\mathrm{bar}}(A)=\{0\}$ for all $n \geqslant 0$;

(iii) $H_{\text {bar }}^{n}(A)=\{0\}$ for all $n \geqslant 0$;

(iv) There exists a long exact Connes-Tsygan sequence of continuous cohomology groups

$$
\cdots \rightarrow H_{\text {naive }}^{n}(A) \rightarrow H C^{n-1}(A) \rightarrow H C^{n+1}(A) \rightarrow H_{\text {naive }}^{n+1}(A) \rightarrow \cdots .
$$

The following propositions are about the equivalence between the continuous cyclic homology of $A$ and the continuous periodic cyclic homology of $A$ when $A$ has trivial continuous Hochschild homology $H H_{n}(A)$ for all $n \geqslant N$ for some integer $N$. They are corollaries of Propositions 2.1 and 2.3, and the above results. For Fréchet algebras, one also needs [20, Lemma 0.5.9] or [2, Lemma 7.1.32].

Proposition 3.2. Let $A$ be a complete locally convex algebra. Then, for any even integer $N$, say $N=2 K$, and the following assertions, we have (i) $)_{N} \Rightarrow(\text { ii) })_{N} \Rightarrow(\text { iii) })_{N} \Rightarrow$ (ii) $N+1$ and (ii) $)_{N} \Rightarrow(\text { iv) })_{N}$ :

(i) $H_{n}^{\text {naive }}(A)=\{0\}$ for all $n \geqslant N$ and $H_{n}^{\text {bar }}(A)=\{0\}$ for all $n \geqslant N-1$;

(ii) $)_{N} H H_{n}(A)=\{0\}$ for all $n \geqslant N$; 
(iii) $_{N}$ for all $k \geqslant K$, up to isomorphism of linear spaces, $H C_{2 k}(A)=H C_{N}(A)$ and $H C_{2 k+1}(A)=H C_{N-1}(A)$;

$(\text { iv })_{N}$ up to isomorphism of linear spaces, $H P_{0}(A)=H C_{N}(A)$ and $H P_{1}(A)=$ $H C_{N-1}(A)$.

For Fréchet algebras, the above isomorphisms are topological isomorphisms.

Proposition 3.3. Let $A$ be a complete locally convex algebra. Then, for any even integer $N$, say $N=2 K$, and the following assertions, we have (i $)_{N} \Rightarrow(\text { ii })_{N} \Rightarrow(\text { iii })_{N} \Rightarrow$ (ii) $)_{N+1}$ and (ii) $)_{N} \Rightarrow(\text { iv })_{N}$ :

(i) ${ }_{N} H_{\text {naive }}^{n}(A)=\{0\}$ for all $n \geqslant N$ and $H_{\text {bar }}^{n}(A)=\{0\}$ for all $n \geqslant N-1$;

$(\text { ii) })_{N}$ for all $n \geqslant N, H H^{n}(A)=\{0\}$;

(iii) $_{N}$ for all $k \geqslant K$, up to isomorphism of linear spaces, $H C^{2 k}(A)=H C^{N}(A)$ and $H C^{2 k+1}(A)=H C^{N-1}(A)$;

(iv) $)_{\mathrm{N}}$ up to isomorphism of linear spaces, $H P^{0}(A)=H C^{N}(A)$ and $H P^{1}(A)=$ $H C^{N-1}(A)$.

\section{Applications to Fréchet and operator algebras}

To start with we recall some notation and terminology used in homological theory. These can be found in any text book on homological algebra (see, for example, MacLane [32] for the algebraic case and Helemskii [20] for the topological case). Furthermore, we apply the results of $\S 3$ and some well-known results from the homology of Banach algebras to calculate continuous cyclic, periodic cyclic and entire cyclic homology and cohomology groups of some Fréchet, Banach and operator algebras. These results will be used in $\S 6$ for the calculation of cyclic-type homology and cohomology of some locally $m$-convex algebras by a consistent use of projective limits of Banach algebras.

\subsection{Homology of topological algebras}

For any Fréchet algebra $A$, not necessarily unital, $A_{+}$is the Fréchet algebra obtained by adjoining an identity to $A$. The linear span of the set $\left\{a_{1} a_{2}: a_{1}, a_{2} \in A\right\}$ is denoted by $A^{2}$, and the closure of $A^{2}$ is denoted by $\overline{A^{2}}$. The space of continuous traces on $A$ is denoted by $A^{\text {tr }}$, that is, $A^{\text {tr }}=\left\{f \in A^{\prime}: f(a b)=f(b a)\right.$ for all $\left.a, b \in A\right\}$. Here, for a Fréchet space $E$, we will denote by $E^{\prime}$ the strong dual space of $E$. The closure in $A$ of the linear span of elements of the form $\{a b-b a: a, b \in A\}$ is denoted by $[A, A]$.

The algebra $A^{\mathrm{e}}=A_{+} \hat{\otimes} A_{+}^{\mathrm{op}}$ is called the enveloping algebra of $A ; A_{+}^{\mathrm{op}}$ is the opposite algebra of $A_{+}$with multiplication $a \cdot b=b a, \hat{\otimes}$ denotes the completed projective tensor product. Every Fréchet $A$-bimodule can be regarded as a unital left Fréchet $A^{\mathrm{e}}$-module.

Let $A$ be a Fréchet algebra and let $X$ be a Fréchet $A$-bimodule. One can define the continuous homology $\mathcal{H}_{n}(A, X)$ of the algebra $A$ with coefficients in $X$ [20, II.5.28], 
and the continuous cohomology group $\mathcal{H}^{n}(A, X)$ of $A$ with coefficients in $X$ [20, Definition I.3.2]. For definition of the $n$th derived functor $\operatorname{Tor}_{n}^{A}(X, Y)$ of $X \hat{\otimes}_{A}$. applied to $Y$, see $[20, \S$ III.4.4]; here $X$ is a Fréchet right $A$-module and $Y$ is a Fréchet left $A$-module.

Recall the definition of $\mathcal{H}_{n}(A, X)$. Let $A$ be a Fréchet algebra and let $X$ be a Fréchet $A$-bimodule. We will define the continuous homology $\mathcal{H}_{n}(A, X)$ of the algebra $A$ with coefficients in $X$ (see, for example, $[\mathbf{2 4}]$ or $\left[\mathbf{2 0}\right.$, II.5.28]). We denote by $C_{n}(A, X), n=$ $0,1, \ldots$, the Fréchet space $X \hat{\otimes} A^{\otimes} n$; we shall call the elements of this space $n$-chains. We also set $C_{0}(A, X)=X$. From the chains we form the standard homology complex

$$
0 \longleftarrow C_{0}(A, X) \stackrel{d_{0}}{\longleftarrow} \cdots \longleftarrow C_{n}(A, X) \stackrel{d_{n}}{\longleftarrow} C_{n+1}(A, X) \longleftarrow \cdots, \quad\left(\mathcal{C}_{\sim}(A, X)\right)
$$

where the differential $d_{n}$ is given by the formula

$$
\begin{aligned}
d_{n}\left(x \otimes a_{1} \otimes a_{2} \otimes \cdots \otimes a_{n+1}\right)=( & \left.\cdot a_{1} \otimes a_{2} \otimes \cdots \otimes a_{n+1}\right) \\
& +\sum_{i=1}^{n}(-1)^{i}\left(x \otimes a_{1} \otimes \cdots \otimes a_{i} a_{i+1} \otimes \cdots \otimes a_{n+1}\right) \\
& +(-1)^{n+1}\left(a_{n+1} \cdot x \otimes a_{1} \otimes \cdots \otimes a_{n}\right) .
\end{aligned}
$$

The $n$th homology group of $\left(\mathcal{C}_{\sim}(A, X)\right)$, denoted by $\mathcal{H}_{n}(A, X)$, is called the $n$th continuous homology group of the Fréchet algebra $A$ with coefficients in $X$.

For Banach algebras there is a well-studied notion of amenability. Recall some definitions. A Banach $A$-bimodule $M=\left(M_{*}\right)^{\prime}$, where $M_{*}$ is a Banach $A$-bimodule, is called dual. A Banach algebra $A$ such that $\mathcal{H}^{n}\left(A, X^{\prime}\right)=\{0\}$ for all $n \geqslant 1$ and for all dual $A$-bimodules $X^{\prime}$ is called amenable [24]. A Banach algebra $A$ is called $N$-amenable if $\mathcal{H}^{n}\left(A, X^{\prime}\right)=\{0\}$ for all $n \geqslant N$ for all dual Banach $A$-bimodule $X^{\prime}($ see $[\mathbf{3 9}, \mathbf{4 0}])$. The weak bidimension of a Banach algebra $A$ is

$$
\operatorname{db}_{w} A=\inf \left\{n: \mathcal{H}^{n+1}\left(A, X^{\prime}\right)=\{0\} \text { for all Banach } A \text {-bimodules } X\right\} .
$$

Note that $\mathcal{H}^{n}\left(A,\left(M_{*}\right)^{\prime}\right)$ can be computed as the $n$th cohomology of the dual complex to $\mathcal{C}_{\sim}\left(A, M_{*}\right)$ (see, for example, [24] or [20, II.5.27]). Therefore, in view of Proposition 2.4 presented above, one can extend the notion of amenability to Fréchet algebras. A Fréchet algebra $A$ such that, for all Fréchet $A$-bimodules $X$ and for all $n \geqslant 1$, the $n$th cohomology of the dual complex of $\left(\mathcal{C}_{\sim}(A, X)\right), H^{n}\left(\mathcal{C}_{\sim}(A, X)^{\prime}\right)=\{0\}$ is called amenable. An equivalent definition is the following. A Fréchet algebra $A$ is amenable if, for all Fréchet $A$-bimodules $X, H_{n}\left(\mathcal{C}_{\sim}(A, X)\right)=\{0\}$ for all $n \geqslant 1$ and $H_{0}\left(\mathcal{C}_{\sim}(A, X)\right)$ is Hausdorff. A Fréchet algebra $A$ is called $N$-amenable if $H^{n}\left(\mathcal{C}_{\sim}(A, X)^{\prime}\right)=\{0\}$ for all $n \geqslant N$ for all Fréchet $A$-bimodules $X$. The weak bidimension of a Fréchet algebra $A$ is

$$
\operatorname{db}_{w} A=\inf \left\{n: H^{n+1}\left(\mathcal{C}_{\sim}(A, X)^{\prime}\right)=\{0\} \text { for all Fréchet } A \text {-bimodules } X\right\} .
$$

Recall that the algebra $A$ is said to be biflat if it is flat in the category of Fréchet $A$-bimodules [20, Definition 7.2.5]. It is known that any amenable Fréchet algebra is biflat $[\mathbf{2 0}]$. 


\subsection{Applications to Fréchet algebras of finite weak bidimension}

The entire cyclic cohomology $H E^{k}(A)$ of $A$ for $k=1,2$ is defined in [9, $\S$ IV.7].

Corollary 4.1. Let $A$ be a Fréchet algebra. Suppose that $\mathrm{db}_{w} \quad A=m$ and $m=2 L$ is an even integer. Then,

(i) for all $\ell \geqslant L, H C_{2 \ell+2}(A)=H C_{m}(A)$ and $H C_{2 \ell+3}(A)=H C_{m+1}(A)$;

(ii) $H P_{0}(A)=H C_{m}(A)$ and $H P_{1}(A)=H C_{m+1}(A)$;

(iii) for all $\ell \geqslant L, H C^{2 \ell+2}(A)=H C^{m}(A)$ and $H C^{2 \ell+3}(A)=H C^{m+1}(A)$;

(iv) $H P^{0}(A)=H C^{m}(A)$ and $H P^{1}(A)=H C^{m+1}(A)$;

(v) if, furthermore, $A$ is a Banach algebra, $H E^{0}(A)=H P^{0}(A)=H C^{m}(A)$ and $H E^{1}(A)=H P^{1}(A)=H C^{m+1}(A)$.

There are similar formulae for odd $m$.

Proof. Since $\mathrm{db}_{w} A=m$, the cohomology groups $H_{\text {naive }}^{n}(A)=H^{n}\left(\mathcal{C}_{\sim}(A, A)^{\prime}\right)=\{0\}$ for all $n \geqslant m+1$ and $H_{\text {bar }}^{n}(A)=H^{n+1}\left(\mathcal{C}_{\sim}(A, \mathbb{C})^{\prime}\right)=\{0\}$ for all $n \geqslant m$; here $\mathbb{C}$ is the trivial $A$-bimodule. Therefore, $H H^{n}(A)=\{0\}$ for all $n \geqslant m+1$. By Proposition 2.4, the homology groups $H H_{n}(A)=\{0\}$ for all $n \geqslant m+1$. One can apply Propositions 3.2 and 3.3 for $N=m+1$ to get relations (i)-(iv) between the continuous cyclic and periodic cyclic homology and cohomology groups for the Fréchet algebra $A$. Regarding (v), by $[\mathbf{2 6}$, Theorem 5.2], for a Banach algebra $A$ of a finite weak bidimension $\mathrm{db}_{w} A$, we have $H E^{k}(A)=H P^{k}(A)$ for $k=0,1$.

Remark 4.2. It is obvious that, for amenable Fréchet algebras $A, \mathrm{db}_{w} A=0$ and, for $N$-amenable Fréchet algebras $A, \mathrm{db}_{w} A \leqslant N-1$. It is known that, for a biflat Banach algebra $A, \mathrm{db}_{w} A \leqslant 2[\mathbf{4 2}$, Theorem 6$]$. Examples of amenable and biflat Banach algebras are presented below in $\S 4.3$. One can also find examples of Banach algebras with $\mathrm{db}_{w} A \geqslant 2$ in [40].

Remark 4.3. Recall that the homological dimension of $A_{+}$in the category of Fréchet $A$-bimodules is called the homological bidimension of $A$. It is denoted by $\mathrm{db} A$ (see [44] or [20, Definition 3.5.9]). If db $A=m$, by [44] or by [20, Theorem 3.4.25], for any Fréchet $A$-bimodule $X$, the homology groups $\mathcal{H}_{n}(A, X)=\operatorname{Tor}_{n}^{A^{\mathrm{e}}}\left(X, A_{+}\right)=\{0\}$ for all $n \geqslant m+1$ and $\mathcal{H}_{m}(A, X)=\operatorname{Tor}_{m}^{A^{\mathrm{e}}}\left(X, A_{+}\right)$is Hausdorff. Hence $\mathrm{db}_{w} \quad A \leqslant \mathrm{db} A=m$ and one can apply Corollary 4.1 .

Example 4.4 (Fréchet algebras of finite homological bidimension).

(i) Let $\mathcal{O}(\mathcal{U})$ be the Fréchet algebra of holomorphic functions on a polydomain $U=$ $U_{1} \times U_{2} \times \cdots U_{m} \subseteq \mathbb{C}^{m}$. Then $\mathrm{db} \mathcal{O}(\mathcal{U})=m[\mathbf{4 5}]$.

(ii) Let $M$ be any infinitely smooth manifold of topological dimension $m$, and let $C^{\infty}(M)$ be the Fréchet algebra of all infinitely smooth functions on $M$. Then $\mathrm{db} C^{\infty}(M)=m[35]$. For relations between the continuous cyclic cohomology

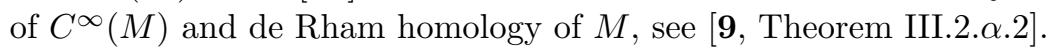


(iii) Let $\mathcal{S}\left(\mathbb{R}^{m}\right)$ be the Fréchet algebra of rapidly decreasing infinitely smooth functions on $\mathbb{R}^{m}$. Then $\mathrm{db} \mathcal{S}\left(\mathbb{R}^{m}\right)=m[\mathbf{3 6}]$.

Hence, for these Fréchet algebras: $\mathcal{O}(\mathcal{U}), C^{\infty}(M)$ and $\mathcal{S}\left(\mathbb{R}^{m}\right)$, the conditions of Corollary 4.1 are satisfied. For example, by Corollary 4.1 and [46, $\S \mathrm{VII}]$, for an even $m$, $H P^{0}\left(\mathcal{S}\left(\mathbb{R}^{m}\right)\right)=H C^{m}\left(\mathcal{S}\left(\mathbb{R}^{m}\right)\right)$, the one-dimensional linear space generated by the fundamental $m$-trace

$$
\phi\left(f^{0}, f^{1}, \ldots, f^{m}\right)=\int_{\mathbb{R}^{m}} f^{0} \mathrm{~d} f^{1} \wedge \cdots \wedge \mathrm{d} f^{m},
$$

and $H P^{1}\left(\mathcal{S}\left(\mathbb{R}^{m}\right)\right)=H C^{m+1}\left(\mathcal{S}\left(\mathbb{R}^{m}\right)\right)=\{0\}$.

\subsection{Applications to biflat Fréchet and Banach algebras}

Let $A$ be a biflat Fréchet algebra. By [20, Proposition 7.1.2 and Theorem 3.4.25], the homology groups $H_{n}^{\text {naive }}(A)=\mathcal{H}_{n}(A, A)=\operatorname{Tor}_{n}^{A^{\mathrm{e}}}\left(A, A_{+}\right)=\{0\}$ for all $n \geqslant 1$. Note that, for the trivial $A$-bimodule $\mathbb{C}$, there is a flat resolution

$$
0 \leftarrow \mathbb{C} \leftarrow A_{+} \leftarrow A \leftarrow 0
$$

in the category of left or right Fréchet $A$-modules. Therefore, by [20, Proposition 7.1.2 and Theorem 3.4.26], the homology groups $H_{n}^{\mathrm{bar}}(A)=\mathcal{H}_{n+1}(A, \mathbb{C})=\operatorname{Tor}_{n+1}^{A}(\mathbb{C}, \mathbb{C})=\{0\}$ for all $n \geqslant 1$. Hence $H H_{n}(A)=\{0\}$ for all $n \geqslant 2$. One can apply Propositions 3.2 and 3.3 for $N=2$ to get relations between continuous cyclic and periodic cyclic homology and cohomology groups for biflat Fréchet algebras.

In the Banach case it is also known that $A=\overline{A^{2}}$ and therefore $H_{0}^{\text {bar }}(A)=\{0\}$. Hence, one obtains the following result.

Corollary 4.5. Let $A$ be a biflat Banach algebra. Then, up to topological isomorphism:

(i) for all $\ell \geqslant 0, H C_{2 \ell}(A)=A /[A, A]$ and $H C_{2 \ell+1}(A)=\{0\}$;

(ii) $H P_{0}(A)=A /[A, A]$ and $H P_{1}(A)=\{0\}$;

(iii) for all $\ell \geqslant 0, H C^{2 \ell}(A)=A^{\text {tr }}$ and $H C^{2 \ell+1}(A)=\{0\}$;

(iv) $H E^{0}(A)=H P^{0}(A)=A^{\text {tr }}$ and $H E^{1}(A)=H P^{1}(A)=\{0\}$.

Proof. Statements (i) and (iii) are shown in [21, Theorem 25 and § 5]. Statements (ii) and (iv) on continuous periodic cyclic homology and cohomology follow from (i), (iii) and Propositions 3.2 and 3.3 for $N=1$. Statement (iv), on entire cyclic cohomology, follows from [26, Theorem 5.2] and [42, Theorem 6]. Recall that, for a biflat Banach algebra $A, \mathrm{db}_{w} A \leqslant 2[\mathbf{4 2}$, Theorem 6]. By [26, Theorem 5.2], for a Banach algebra $A$ of a finite weak bidimension $\operatorname{db}_{w} A$, we have $H E^{k}(A)=H P^{k}(A)$ for $k=0,1$. In view of [20, Lemma 0.5.9] or [2, Lemma 7.1.32], one can deduce that these isomorphisms are topological isomorphisms. 


\section{Example 4.6 (biflat Banach algebras).}

(i) Amenable Banach algebras. Each nuclear $C^{*}$-algebra is amenable [18]. Some examples of amenable $C^{*}$-algebras are: (a) GCR $C^{*}$-algebras (in particular, commutative $C^{*}$-algebras and the $C^{*}$-algebra of compact operators $\mathcal{K}(H)$ on a Hilbert space $H$ ); (b) uniformly hyperfinite algebras [34, Remark 6.2.4].

Let $G$ be a locally compact group with a left-invariant Haar measure $d s$. The group algebra $L^{1}(G)$ of Haar integrable functions on an amenable locally compact group $G$ with convolution product is also amenable [24]. Therefore, by Corollary 4.5, for the continuous cyclic and periodic cyclic cohomology groups and the entire cyclic cohomology of $L^{1}(G)$, we have the following. For all $\ell \geqslant 0$,

$$
H E^{0}\left(L^{1}(G)\right)=H P^{0}\left(L^{1}(G)\right)=H C^{2 \ell}\left(L^{1}(G)\right)=L^{1}(G)^{\operatorname{tr}},
$$

where $L^{1}(G)^{\operatorname{tr}}=\left\{f \in L^{\infty}(G): f(a b)=f(b a)\right.$ for all $\left.a, b \in L^{1}(G)\right\}$ and

$$
H E^{1}\left(L^{1}(G)\right)=H P^{1}\left(L^{1}(G)\right)=H C^{2 \ell+1}\left(L^{1}(G)\right)=\{0\} .
$$

It is shown in $[\mathbf{1 7}]$ that the Banach algebra $\mathcal{K}(E)$ of compact operators on a Banach space $E$ with property $(\boldsymbol{A})$ which was defined in $[\mathbf{1 7}]$ is amenable. Property $(\boldsymbol{A})$ implies that $\mathcal{K}(E)$ contains a bounded sequence of projections of unbounded finite rank, and from this it is easy to show (via embedding of matrix algebras) that there is no non-zero bounded trace on $\mathcal{K}(E)$. Therefore, by Corollary 4.5 , the continuous cyclic and periodic cyclic homology and cohomology groups and the entire cyclic cohomology of $\mathcal{K}(E)$ are trivial, that is, for all $n \geqslant 0$,

$$
H C_{n}(\mathcal{K}(E))=\{0\}, \quad H C^{n}(\mathcal{K}(E))=\{0\},
$$

and, for $k=0,1$,

$$
H P_{k}(\mathcal{K}(E))=\{0\}, \quad H E^{k}(\mathcal{K}(E))=H P^{k}(\mathcal{K}(E))=\{0\} .
$$

Further examples of amenable Banach algebras can be found in [17].

(ii) Biprojective Banach algebras. The definition and examples of biprojective Banach algebras are given in [20, $\S$ IV.5]. One example is the Banach algebra $\ell_{1}$ of summable complex sequences $\left(\xi_{n}\right)$ with coordinatewise operations. By Corollary 4.5 , for all $k \geqslant 0$,

$$
\begin{aligned}
H P_{0}\left(\ell_{1}\right)= & H C_{2 k}\left(\ell_{1}\right)=\ell_{1}, \quad H P_{1}\left(\ell_{1}\right)=H C_{2 k+1}\left(\ell_{1}\right)=\{0\}, \\
& H E^{0}\left(\ell_{1}\right)=H P^{0}\left(\ell_{1}\right)=H C^{2 k}\left(\ell_{1}\right)=\ell_{\infty},
\end{aligned}
$$

where $\ell_{\infty}$ is the Banach space of bounded sequences, and

$$
H E^{1}\left(\ell_{1}\right)=H P^{1}\left(\ell_{1}\right)=H C^{2 k+1}\left(\ell_{1}\right)=\{0\} .
$$

(iii) The algebra $\mathcal{K}\left(\ell_{2} \hat{\otimes} \ell_{2}\right)$ of all compact operators on the Banach space $\ell_{2} \hat{\otimes} \ell_{2}$ provides an example of a non-amenable, biflat semisimple Banach algebra with a left-bounded approximate identity $[\mathbf{4 3}]$. 


\subsection{Applications to Fréchet and operator algebras with trivial $H H_{n}(A)$ for all $n \geqslant 0$}

Corollary 4.7. Let $A$ be a Fréchet algebra. Suppose that $H H_{n}(A)=\{0\}$ for all $n \geqslant 0$. Then

$$
H H^{n}(A)=H C_{n}(A)=H C^{n}(A)=\{0\} \quad \text { for all } n \geqslant 0,
$$

and

$$
H P_{k}(A)=H P^{k}(A)=\{0\} \quad \text { for } k=0,1
$$

Proof. By [5, Lemma 2], $H H_{n}(A)=\{0\}$ for all $n \geqslant 0$ if and only if $H H^{n}(A)=\{0\}$ for all $n \geqslant 0$. Hence, by [5, Lemmas 1 and 2], $H C_{n}(A)=H C^{n}(A)=\{0\}$ for all $n \geqslant 0$ and $H P_{k}(A)=H P^{k}(A)=\{0\}$ for $k=0,1$.

Corollary 4.8. Let $A$ be a $C^{*}$-algebra without non-zero bounded traces. Then

$$
H H_{n}(A)=H H^{n}(A)=H C_{n}(A)=H C^{n}(A)=\{0\} \quad \text { for all } n \geqslant 0,
$$

and

$$
H P_{k}(A)=H P^{k}(A)=\{0\} \quad \text { for } k=0,1
$$

Proof. By [12, Proposition 1.7.3], any $C^{*}$-algebra has a bounded approximate identity and so $H_{\text {bar }}^{n}(A)=\{0\}$ for all $n \geqslant 0[\mathbf{2 4}]$. By [8, Theorem 4.1 and Corollary 3.3], for every $C^{*}$-algebras without non-zero bounded traces, the simplicial cohomology groups $H_{\text {naive }}^{n}(A)=\mathcal{H}^{n}\left(A, A^{*}\right)=\{0\}$ for all $n \geqslant 0$. Therefore, $H H^{n}(A)=\{0\}$ for all $n \geqslant 0$. By [5, Lemma 2], $H H^{n}(A)=\{0\}$ for all $n \geqslant 0$. Hence, Corollary 4.7 shows that $H C_{n}(A)=H C^{n}(A)=\{0\}$ for all $n \geqslant 0$ and $H P_{k}(A)=H P^{k}(A)=\{0\}$ for $k=0,1$.

\section{Example 4.9 ( $C^{*}$-algebras without non-zero bounded traces).}

(i) The $C^{*}$-algebra $\mathcal{K}(H)$ of compact operators on an infinite-dimensional Hilbert space $H\left[\mathbf{1}\right.$, Theorem 2]. We can also show that $C(\Omega, \mathcal{K}(H))^{\operatorname{tr}}=\{0\}$, where $\Omega$ is a compact space.

(ii) Properly infinite von Neumann algebras $\mathcal{U}$. By [41, Proposition 2.2.4] in $\mathcal{U}$ there exists a sequence $\left(p_{m}\right)$ of mutually orthogonal, equivalent projections with $p_{m} \sim e$. Thus, by [15, Theorem 2.1], each Hermitian element of $\mathcal{U}$ is the sum of five commutators. Hence, there are no non-zero traces on $\mathcal{U}$. This class includes the $C^{*}$-algebra $\mathcal{B}(H)$ of all bounded operators on an infinite-dimensional Hilbert space $H$; see also [19] for the statement $\mathcal{B}(H)^{\operatorname{tr}}=\{0\}$.

One can see that, for these $C^{*}$-algebras: $\mathcal{K}(H), C(\Omega, \mathcal{K}(H)), \mathcal{B}(H)$, where $H$ is an infinite-dimensional Hilbert space and properly infinite von Neumann algebras $\mathcal{U}$, the conditions of Corollary 4.8 are satisfied. Therefore, for these algebras, the following continuous homology and cohomology groups are trivial: $H H, H C$ and $H P$. 


\section{Cohomology of projective limits of locally convex algebras}

In the first part of this section we recall some notation and terminology used in locally convex spaces and in topological algebras, and we give some necessary explanations. One can consult the books by Mallios [33] on topological algebras and by Jarchow [23] or Köthe $[\mathbf{2 7}, \mathbf{2 8}]$ on locally convex spaces. In the second part of this section we prove the main results of the paper on cohomology and homology of projective limits of locally convex and Fréchet algebras.

\subsection{Inductive and projective limits: definitions, notation and explanations}

We say that an index set $(\Lambda, \leqslant)$ is directed if it is a partially ordered set with respect to $\leqslant$ such that for any indices $\alpha, \beta$ in $\Lambda$ there exists an index $\gamma \in \Lambda$ with $\alpha \leqslant \gamma$ and $\beta \leqslant \gamma$. A subset $\Lambda_{0}$ of $\Lambda$ is called cofinal if for every $\beta$ in $\Lambda$ there is $\alpha$ in $\Lambda_{0}$ such that $\beta \leqslant \alpha$. The definition of an inductive system $\left(E_{\alpha}, S_{\alpha, \beta}\right)^{(\Lambda, \leqslant)}$ of topological vector spaces can be found in $[\mathbf{2 3}, \S 4.5]$. Let $I_{\alpha}: E_{\alpha} \rightarrow \bigoplus_{\beta \in \Lambda} E_{\beta}, \alpha \in \Lambda$, be the canonical injections. Let $L$ be the linear span of the union of all $\operatorname{Im}\left(I_{\alpha}-I_{\beta} \circ S_{\beta, \alpha}\right)$, where $\alpha, \beta \in \Lambda$ such that $\alpha \leqslant \beta$. Then the quotient space $\left(\bigoplus_{\beta \in \Lambda} E_{\beta}\right) / L$, endowed with the quotient topology, is called the topological inductive limit of $\left(E_{\alpha}, S_{\alpha, \beta}\right)^{(\Lambda, \leqslant)}$. We denote the inductive limit by $\lim _{\vec{\alpha}} E_{\alpha}$ or $\operatorname{ind}_{\alpha \in \Lambda} E_{\alpha}$ (see, for example, $[\mathbf{2 3}, \S 4.5]$ ). Note that on ignoring the topology in $\lim _{\vec{\alpha}} E_{\alpha}$ we obtain the algebraic inductive limit.

Let $Q: \bigoplus_{\beta \in \Lambda} E_{\beta} \rightarrow \lim _{\vec{\alpha}} E_{\alpha}$ be the quotient map corresponding to our construction and let, by definition, $S_{\beta}=Q \circ I_{\beta}, \beta \in \Lambda$. Recall that an inductive system $\left(E_{\alpha}, S_{\alpha, \beta}\right)^{(\Lambda, \leqslant)}$ of topological vector spaces, as well as the corresponding inductive limit, is said to be reduced if the maps

$$
S_{\beta}: E_{\beta} \rightarrow \lim _{\vec{\alpha}} E_{\alpha}
$$

are injective for every $\beta \in \Lambda$. For a reduced inductive system $\left(E_{\alpha}, S_{\alpha, \beta}\right)^{(\Lambda, \leqslant)}$, as a linear space, $\lim _{\vec{\alpha}} E_{\alpha}$ is just the union $\bigcup_{\alpha \in \Lambda} E_{\alpha}[\mathbf{2 3}, \S 4.5]$.

In the case where $(\Lambda, \leqslant)$ is $\mathbb{N}$ with its natural order, a reduced inductive system $\left(E_{m}, S_{m, n}\right)^{\mathbb{N}}$ of topological vector spaces, and the corresponding inductive limit, are said to be strict if the topological vector space $E_{n}$ is a subspace of $E_{m}$ and $S_{m, n}$ is the embedding of $E_{n}$ into $E_{m}$ for $n \leqslant m$ in $\mathbb{N}$ and the topology of $E_{n}$ is equal to the one induced by $E_{m}$.

The definition of a projective system $\left(E_{\alpha}, T_{\alpha, \beta}\right)_{(\Lambda, \leqslant)}$ of topological vector spaces can be found in $[\mathbf{2 3}, \S 2.6]$. The subspace $F$ of $\prod_{\alpha \in \Lambda} E_{\alpha}$ which consists of all $\left(x_{\alpha}\right) \in \prod_{\alpha \in \Lambda} E_{\alpha}$ such that $T_{\alpha, \beta} x_{\beta}=x_{\alpha}$ holds for $\alpha \leqslant \beta$ in $\Lambda$ is called the projective limit of the projective system. We always consider $F$ as a topological vector space with respect to the relative topology of the product. We denote the projective limit by $\lim _{\overleftarrow{\alpha}} E_{\alpha} \operatorname{or}_{\operatorname{Proj}_{\alpha \in \Lambda}} E_{\alpha}$ (see, for example, $[\mathbf{2 3}, \S 2.6])$. On ignoring the topology in $\lim _{\overleftarrow{\alpha}} E_{\alpha}$, we obtain the algebraic projective limit.

Define $T_{\beta}$ as the restrictions of the projection $\operatorname{Pr}_{\beta}: \prod_{\alpha \in \Lambda} E_{\alpha} \rightarrow E_{\beta}, \beta \in \Lambda$, on $\lim _{\overleftarrow{\alpha}} E_{\alpha}$. Note that $T_{\alpha}=T_{\alpha, \beta} \circ T_{\beta}$ if $\alpha \leqslant \beta$. Recall that a projective system $\left(E_{\alpha}, T_{\alpha, \beta}\right)_{(\Lambda, \leqslant)}$ of topological vector spaces, as well as the corresponding projective limit, is said to be 
reduced if the maps

$$
T_{\beta}: \lim _{\overleftarrow{\alpha}} E_{\alpha} \rightarrow E_{\beta}
$$

have dense range for every $\beta \in \Lambda[\mathbf{2 3}, \S 2.6]$.

One can find the definition of the topological derived functors

$$
\lim _{\overleftarrow{j}} n, \quad n \geqslant 0,
$$

of $\lim _{\leftarrow}$ in $[\mathbf{3 7}]$ or $[\mathbf{1 4}, \S 3.2]$. Recall that one possible construction of the derived functor $\lim _{\leftarrow}^{1}$ is as follows (see $[\mathbf{3 7}, \S 5]$ or $[\mathbf{1 4}, \S 3.2]$ ). Firstly, every projective system of Fréchet spaces $\left(E_{j}, T_{j, k}\right)_{(\mathbb{N}, \leqslant)}$ possesses a free resolution of the form

$$
0 \rightarrow\left(E_{j}\right)_{j \in \mathbb{N}} \stackrel{u}{\rightarrow}\left(L_{j}\right)_{j \in \mathbb{N}} \stackrel{v}{\rightarrow}\left(L_{j-1}\right)_{j \in \mathbb{N}} \rightarrow 0,
$$

where $L_{0}=\{0\}$ and, for all $j \geqslant 1, L_{j}=\prod_{k=1}^{j} E_{k}$ is the free projective system with generators $E_{1}, \ldots, E_{k}, \ldots$, and the morphisms $u=\left(u_{j}\right)$ and $v=\left(v_{j}\right)$ of projective systems are defined by the formulae

$$
u_{j}: E_{j} \rightarrow L_{j}: x \mapsto\left(T_{1, j} x, \ldots, T_{j, j} x\right)
$$

and

$$
v_{j}: L_{j} \rightarrow L_{j-1}:\left(x_{1}, \ldots, x_{j}\right) \mapsto\left(x_{1}-T_{1,2} x_{2}, \ldots, x_{j-1}-T_{j-1, j} x_{j}\right) \quad\left(x_{j} \in E_{j}\right) .
$$

Since the projective limit is a left-exact functor, the short exact sequence (5.1) induces a sequence of Fréchet spaces and continuous operators

$$
0 \longrightarrow \lim _{\overleftarrow{j}} E_{j} \stackrel{\operatorname{Proj}(u)}{\longrightarrow} \prod_{j \in \mathbb{N}} E_{j} \stackrel{\operatorname{Proj}(v)}{\longrightarrow} \prod_{j \in \mathbb{N}} E_{j}
$$

which is left exact. Then one can deduce that

$$
\lim _{\overleftarrow{j}}^{1} E_{j}=\operatorname{Coker} \operatorname{Proj}(v) \text { and } \lim _{\overleftarrow{j}}^{n} E_{j}=\{0\} \quad \text { for } n \geqslant 2
$$

Therefore, on ignoring the topology in $\lim _{\leftarrow} E_{j} E_{j}$, we obtain the algebraic $\lim _{\overleftarrow{j}}^{1} E_{j}$ (see $[\mathbf{4 7}, \S 3.5]$ for a similar construction in the algebraic case).

Recall that a projective system $\left(E_{j}, T_{j, k}\right)_{(\mathbb{N}, \leqslant)}$ with the property that all structural maps $T_{j, k}: E_{k} \rightarrow E_{j}, j \leqslant k$, have dense range is called a Mittag-Leffler system [14, Definition 3.2.10]. One should be careful not to confuse this definition with the purely algebraic version [47, Definition 3.5.6].

A projective system $\left(E_{j}, T_{j, k}\right)_{(\mathbb{N}, \leqslant)}$ of Fréchet spaces is a Mittag-Leffler system if and only if it is reduced (see, for example, [3, Chapitre II, $\S 3$, Théorème 1]). Therefore, by [14, Theorem 3.2.4 and Lemma 3.2.3], for a reduced projective system $\left(E_{j}, T_{j, k}\right)_{(\mathbb{N}, \leqslant)}$, $\lim _{\leftarrow}^{1} E_{j}=\{0\}$.

A projective system of topological algebras is, by definition, a projective system

$$
\left(A_{\alpha}, T_{\alpha, \beta}\right)_{(\Lambda, \leqslant)}
$$


such that $A_{\alpha}, \alpha \in \Lambda$, are topological algebras and $T_{\alpha, \beta}$, with $\alpha \leqslant \beta$ in $\Lambda$, are continuous morphisms of algebras [33, Definition III.2.1]. The projective limit $A=\lim _{\overleftarrow{\alpha}} A_{\alpha}$ is a closed subalgebra of the Cartesian product topological algebra $\prod_{\alpha \in \Lambda} A_{\alpha}$. In particular, $A$ is complete if each $A_{\alpha}, \alpha \in \Lambda$, is complete; $A$ is Hausdorff if each $A_{\alpha}, \alpha \in \Lambda$, is Hausdorff. The projective $\operatorname{limit} A=\lim _{\overleftarrow{\alpha}} A_{\alpha}$ of locally convex algebras with jointly continuous multiplications is a topological algebra of the same type [33, $\S \S$ III.1, 2].

\subsection{Main results on cohomology of projective limits of locally convex algebras}

Theorem 5.1. Let $\left(A_{\alpha}, T_{\alpha, \beta}\right)_{(\Lambda, \leqslant)}$ be a reduced projective system of complete Hausdorff locally convex algebras with jointly continuous multiplications, and let $A$ be the projective limit algebra $A=\lim _{\overleftarrow{\alpha}} A_{\alpha}$. Then, up to isomorphism of linear spaces, for all $n \geqslant 0$,

$$
H C^{n}(A)=\lim _{\vec{\alpha}} H C^{n}\left(A_{\alpha}\right)
$$

and, for $k=0,1$,

$$
H P^{k}(A)=\lim _{\vec{\alpha}} H P^{k}\left(A_{\alpha}\right) .
$$

The same is true for the following cohomology groups: $H_{\text {naive }}, H_{\text {bar }}$ and $H H$.

Proof. We deduce the theorem from a sequence of lemmas.

Lemma 5.2. Let $\left(A_{\alpha}, T_{\alpha, \beta}\right)_{(\Lambda, \leqslant)}$ be a reduced projective system of complete Hausdorff locally convex algebras with jointly continuous multiplications and let $A=\lim _{\overleftarrow{\alpha}} A_{\alpha}$. Then

(i) for all $n \geqslant 1,\left(A_{\alpha}^{\hat{\otimes} n}, T_{\alpha, \beta}^{\hat{\otimes} n}\right)_{(\Lambda, \leqslant)}$ is a reduced projective system of complete Hausdorff locally convex algebras,

(ii) for all $n \geqslant 0$, up to topological isomorphism,

$$
\mathcal{C}_{n}(A) \stackrel{\text { def }}{=} A^{\hat{\otimes}(n+1)}=\lim _{\overleftarrow{\alpha}} A_{\alpha}^{\hat{\otimes}(n+1)},
$$

where the isomorphism is the linear continuous extension of

$$
\left(a_{\alpha_{1}}^{(1)}\right)_{\alpha_{1}} \otimes\left(a_{\alpha_{2}}^{(2)}\right)_{\alpha_{2}} \otimes \cdots \otimes\left(a_{\alpha_{n+1}}^{(n+1)}\right)_{\alpha_{n+1}} \mapsto\left(a_{\alpha}^{(1)} \otimes a_{\alpha}^{(2)} \otimes \cdots \otimes a_{\alpha}^{(n+1)}\right)_{\alpha},
$$

(iii) for all $n \geqslant 1$, the dual system

$$
\left(\left(A_{\alpha}^{\hat{\otimes} n}\right)^{\prime},\left(T_{\alpha, \beta}^{\hat{\otimes} n}\right)^{*}\right)^{(\Lambda, \leqslant)}
$$

is a reduced inductive system and, up to isomorphism of linear spaces,

$$
\mathcal{C}^{n}(A) \stackrel{\text { def }}{=}\left(A^{\hat{\otimes}(n+1)}\right)^{\prime}=\lim _{\vec{\alpha}}\left(A_{\alpha}^{\hat{\otimes}(n+1)}\right)^{\prime}
$$


Proof. (i), (ii) By virtue of properties of the projective tensor product of Hausdorff locally convex spaces $[\mathbf{2 3}, \S \S$ III.15.1, 2] and by [33, Lemma X.3.1], for any $\beta, \alpha \in \Lambda$, $B_{\beta} \hat{\otimes} A_{\alpha}$ is a complete Hausdorff locally convex algebra. In view of $[\mathbf{2 8}, \S 41.6]$ or $[\mathbf{2 3}$, $\S 15.4]$, the projective system $\left(B_{\alpha} \hat{\otimes} A_{\alpha}, F_{\alpha, \beta} \otimes T_{\alpha, \beta}\right)_{(\Lambda, \leqslant)}$ is reduced and, up to topological isomorphism,

$$
B \hat{\otimes} A=\lim _{(\overleftarrow{\beta, \alpha})} B_{\beta} \hat{\otimes} A_{\alpha},
$$

where $(\beta, \alpha) \in(\Lambda \times \Lambda, \leqslant)$ and $(\Lambda \times \Lambda, \leqslant)$ is directed by the order relation: $(\beta, \alpha) \leqslant\left(\beta^{\prime}, \alpha^{\prime}\right)$ if and only if $\beta \leqslant \beta^{\prime}$ and $\alpha \leqslant \alpha^{\prime}$. Note that the directed subset $\{(\alpha, \alpha) \mid \alpha \in(\Lambda, \leqslant)\}$ is cofinal in the directed set $(\Lambda \times \Lambda, \leqslant)$. Therefore, up to topological isomorphism,

$$
\lim _{(\overleftarrow{\beta, \gamma})} B_{\beta} \hat{\otimes} A_{\gamma}=\lim _{\overleftarrow{\alpha}} B_{\alpha} \hat{\otimes} A_{\alpha}
$$

where the isomorphism is given by

$$
\left(w_{(\beta, \gamma)}\right)_{(\beta, \gamma)} \mapsto\left(w_{(\alpha, \alpha)}\right)_{\alpha} .
$$

By induction on $n$, one can see that, for all $n \geqslant 1,\left(A_{\alpha}^{\hat{\otimes} n}, T_{\alpha, \beta}^{\hat{\otimes} n}\right)_{(\Lambda, \leqslant)}$ is a reduced projective system of complete Hausdorff locally convex algebras and, for all $n \geqslant 0$,

$$
\mathcal{C}_{n}(A)=A^{\hat{\otimes}(n+1)}=A^{\hat{\otimes} n} \hat{\otimes} A=\left(\lim _{\overleftarrow{\beta}} A_{\beta}^{\hat{\otimes} n}\right) \hat{\otimes}\left(\lim _{\overleftarrow{\gamma}} A_{\gamma}\right)=\lim _{\overleftarrow{\alpha}} A_{\alpha}^{\hat{\otimes}(n+1)} .
$$

(iii) By [23, Proposition 8.8.7], the dual system of

$$
\left(A_{\alpha}^{\hat{\otimes} n}, T_{\alpha, \beta}^{\hat{\otimes} n}\right)_{(\Lambda, \leqslant)},
$$

that is,

$$
\left(\left(A_{\alpha}^{\hat{\otimes} n}\right)^{\prime},\left(T_{\alpha, \beta}^{\hat{\otimes} n}\right)^{*}\right)^{(\Lambda, \leqslant)}
$$

is a reduced inductive system, and the dual of $\lim _{\overleftarrow{\alpha}} A_{\alpha}^{\hat{\otimes}(n+1)}$ can be identified as a linear space with the inductive limit $\lim _{\vec{\alpha}}\left(A_{\alpha}^{\hat{\otimes}(n+1)}\right)^{\prime}$. Therefore, by part (ii), up to isomorphism of linear spaces,

$$
\mathcal{C}^{n}(A) \stackrel{\text { def }}{=}\left(A^{\hat{\otimes}(n+1)}\right)^{\prime}=\lim _{\vec{\alpha}}\left(A_{\alpha}^{\hat{\otimes}(n+1)}\right)^{\prime} .
$$

Since $\left(\left(A_{\alpha}^{\hat{\otimes} n}\right)^{\prime},\left(T_{\alpha, \beta}^{\hat{\otimes} n}\right)^{*}\right)^{(\Lambda, \leqslant)}$ is a reduced inductive system, as a linear space,

$$
\lim _{\vec{\alpha}}\left(A_{\alpha}^{\hat{\otimes}(n+1)}\right)^{\prime}=\bigcup_{\alpha \in \Lambda}\left(A_{\alpha}^{\hat{\otimes}(n+1)}\right)^{\prime}
$$

(see $[\mathbf{2 3}, \S 4.5])$. Note that the isomorphism

$$
\bigcup_{\alpha \in \Lambda}\left(A_{\alpha}^{\hat{\otimes}(n+1)}\right)^{\prime} \rightarrow\left(A^{\hat{\otimes}(n+1)}\right)^{\prime}
$$

is given by the following map: for each $\alpha \in \Lambda$,

$$
\left(A_{\alpha}^{\hat{\otimes}(n+1)}\right)^{\prime} \rightarrow\left(A^{\hat{\otimes}(n+1)}\right)^{\prime}: f \mapsto f \circ T_{\alpha}^{\hat{\otimes}(n+1)} .
$$


Lemma 5.3. Let $\left(A_{\alpha}, T_{\alpha, \beta}\right)_{(\Lambda, \leqslant)}$ be a reduced projective system of complete Hausdorff locally convex spaces. Then, up to topological isomorphism,

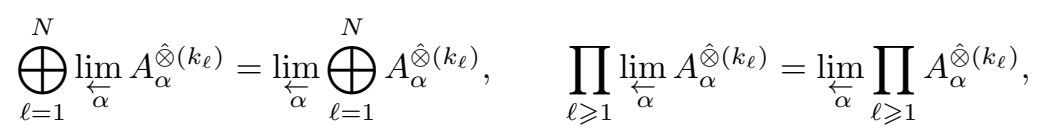

and $\left(\prod_{\ell \geqslant 1} A_{\alpha}^{\hat{\otimes}\left(k_{\ell}\right)},\left(T_{\alpha, \beta}^{\hat{\otimes}\left(k_{\ell}\right)}\right)_{\ell}\right)_{(\Lambda, \leqslant)}$ is a reduced projective system of complete Hausdorff locally convex spaces.

Proof. By Lemma 5.2 and by virtue of properties of topological products [27, $\S \S 1.8$ and 18.3],

$$
\left(\prod_{\ell \geqslant 1} A_{\alpha}^{\hat{\otimes}\left(k_{\ell}\right)},\left(T_{\alpha, \beta}^{\hat{\otimes}\left(k_{\ell}\right)}\right)_{\ell}\right)_{(\Lambda, \leqslant)}
$$

is a reduced projective system of complete Hausdorff locally convex spaces.

The isomorphism

$$
\prod_{\ell \geqslant 1} \lim _{\overleftarrow{\alpha}} A_{\alpha}^{\hat{\otimes}\left(k_{\ell}\right)}=\lim _{\overleftarrow{\alpha}} \prod_{\ell \geqslant 1} A_{\alpha}^{\hat{\otimes}\left(k_{\ell}\right)}
$$

is given by the following map. Let $\left(v_{\alpha}\right)_{\alpha} \in \lim _{\overleftarrow{\alpha}} \prod_{\ell \geqslant 1} A_{\alpha}^{\hat{\otimes}\left(k_{\ell}\right)}$, that is,

$$
v_{\alpha}=\left(v_{\alpha}^{1}, v_{\alpha}^{2}, \ldots, v_{\alpha}^{\ell}, \ldots\right),
$$

where $v_{\alpha}^{\ell} \in A_{\alpha}^{\hat{\otimes}\left(k_{\ell}\right)}$ and for all $\ell \geqslant 0$ we have $T_{\alpha, \beta}^{\hat{\otimes}\left(k_{\ell}\right)} v_{\beta}^{\ell}=v_{\alpha}^{\ell}$ whenever $\alpha, \beta \in \Lambda$ are such that $\alpha \leqslant \beta$. It is clear that, for every $\ell \geqslant 0,\left(v_{\alpha}^{\ell}\right)_{\alpha}$ belongs to $\lim _{\overleftarrow{\alpha}} A_{\alpha}^{\hat{\otimes}\left(k_{\ell}\right)}$. Therefore, the isomorphism can be given by

$$
\left(\left(v_{\alpha}^{\ell}\right)_{\ell=1}^{\infty}\right)_{\alpha} \mapsto\left(\left(v_{\alpha}^{\ell}\right)_{\alpha}\right)_{\ell=1}^{\infty} .
$$

The proof for the direct sum is still simpler.

Conclusion of the proof of Theorem 5.1. Let us prove the result for $H C^{*}$. By definition, $H C^{n}(A)=H^{n}\left(\left(\mathcal{B}_{\mathrm{c}} \bar{\Omega} A_{+}\right)^{\prime}, \tilde{b}^{*}+\tilde{B}^{*}\right)$, where

$$
\begin{aligned}
\left(\mathcal{B}_{\mathrm{c}} \bar{\Omega} A_{+}\right)_{0}= & A, \ldots, \\
& \left(\mathcal{B}_{\mathrm{c}} \bar{\Omega} A_{+}\right)_{2 k-1}=\left(A^{\hat{\otimes} 2} \oplus A\right) \oplus\left(A^{\hat{\otimes} 4} \oplus A^{\hat{\otimes} 3}\right) \oplus \cdots \oplus\left(A^{\hat{\otimes}(2 k)} \oplus A^{\hat{\otimes}(2 k-1)}\right)
\end{aligned}
$$

and

$$
\left(\mathcal{B}_{\mathrm{c}} \bar{\Omega} A_{+}\right)_{2 k}=A \oplus\left(A^{\hat{\otimes} 3} \oplus A^{\hat{\otimes} 2}\right) \oplus \cdots \oplus\left(A^{\hat{\otimes}(2 k+1)} \oplus A^{\hat{\otimes}(2 k)}\right) .
$$

By Lemma 5.3, one can see that

$$
\left(\mathcal{B}_{\mathrm{C}} \bar{\Omega} A_{+}\right)_{2 k-1}=\lim _{\overleftarrow{\alpha}}\left(\left(A_{\alpha}^{\hat{\otimes} 2} \oplus A_{\alpha}\right) \oplus\left(A_{\alpha}^{\hat{\otimes} 4} \oplus A_{\alpha}^{\hat{\otimes} 3}\right) \oplus \cdots \oplus\left(A_{\alpha}^{\hat{\otimes}(2 k)} \oplus A_{\alpha}^{\hat{\otimes}(2 k-1)}\right)\right)
$$

and

$$
\left(\mathcal{B}_{\mathrm{c}} \bar{\Omega} A_{+}\right)_{2 k}=\lim _{\overleftarrow{\alpha}}\left(A_{\alpha} \oplus\left(A_{\alpha}^{\hat{\otimes} 3} \oplus A_{\alpha}^{\hat{\otimes} 2}\right) \oplus \cdots \oplus\left(A_{\alpha}^{\hat{\otimes}(2 k+1)} \oplus A_{\alpha}^{\hat{\otimes}(2 k)}\right)\right) .
$$


In view of Lemma 5.2 , the cochain complex $\left(\left(\mathcal{B}_{\mathrm{c}} \bar{\Omega} A_{+}\right)^{\prime}, \tilde{b}^{*}+\tilde{B}^{*}\right)$ is an inductive limit of the reduced inductive system of cochain complexes $\left(\left(\mathcal{B}_{\mathrm{c}} \bar{\Omega}\left(A_{\alpha}\right)_{+}\right)^{\prime}, \tilde{b}^{*}+\tilde{B}^{*}\right)$. Using the fact that cohomology commutes with inductive limits (see [4, Proposition 1, p. 28] or [7, Proposition V.9.3]), we have

$$
H C^{n}(A)=\lim _{\vec{\alpha}} H C^{n}\left(A_{\alpha}\right) .
$$

The same method works for the cohomology groups $H_{\text {naive }}^{*}, H_{\text {bar }}^{*}, H H^{*}$ and $H P^{*}$.

Theorem 5.4. Let $\left(A_{j}, T_{j, k}\right)_{(J, \leqslant)}$ be a countable reduced projective system of Fréchet algebras, and let $A$ be the projective limit of $\left(A_{j}, T_{j, k}\right)_{(J, \leqslant)}, A=\lim _{\leftarrow} A_{i}$. Then, for all $n \geqslant 0$, the following short sequences

$$
0 \rightarrow \lim _{\overleftarrow{i}}^{1} H C_{n+1}\left(A_{i}\right) \rightarrow H C_{n}(A) \rightarrow \lim _{\overleftarrow{i}} H C_{n}\left(A_{i}\right) \rightarrow 0
$$

and

$$
0 \rightarrow \lim _{\overleftarrow{i}}^{1} H P_{k+1}\left(A_{i}\right) \rightarrow H P_{k}(A) \rightarrow \lim _{\overleftarrow{i}} H P_{k}\left(A_{i}\right) \rightarrow 0, \quad \text { for } k=0,1
$$

are exact. The same statement is true for the following homology groups: $H^{\text {naive }}, H^{\text {bar }}$ and $H H$.

Proof. By Lemmas 5.2 and 5.3, the chain complex $\left(\mathcal{B}_{\mathrm{p}} \bar{\Omega} A_{+}, \tilde{b}+\tilde{B}\right)$ is the projective limit of a reduced projective system of chain complexes $\left(\mathcal{B}_{\mathrm{p}} \bar{\Omega}\left(A_{i}\right)_{+}, \tilde{b}+\tilde{B}\right)$. Since the system is reduced, and therefore is a Mittag-Leffler system of topological spaces, by [14, Theorems 3.2.8 and 3.2.4] and $[\mathbf{3 7}, \S 5]$, we find that

$$
\underset{\leftarrow}{\lim _{i}^{1}}\left(\mathcal{B}_{\mathrm{p}} \bar{\Omega}\left(A_{i}\right)_{+}\right)_{\mathrm{ev}}=\{0\} \quad \text { and } \quad \lim _{\overleftarrow{i}}^{1}\left(\mathcal{B}_{\mathrm{p}} \bar{\Omega}\left(A_{i}\right)_{+}\right)_{\text {odd }}=\{0\}
$$

see $\S 5.1$ for a more detailed explanation. By [47, Theorem 3.5.8], for any tower of chain complexes $\left\{C_{i}\right\}$ and for $C=\lim _{\overleftarrow{i}} C_{i}$ such that $\lim _{\overleftarrow{i}}^{1} C_{i}=\{0\}$, there is an exact sequence for each $n$ :

$$
0 \rightarrow \lim _{i}^{1} H_{n+1}\left(C_{i}\right) \rightarrow H_{n}(C) \rightarrow \lim _{i} H_{n}\left(C_{i}\right) \rightarrow 0 .
$$

Therefore, for the tower of chain complexes $\left\{\mathcal{B}_{\mathrm{p}} \bar{\Omega}\left(A_{i}\right)_{+}, \tilde{b}+\tilde{B}\right\}$ and for

$$
\left(\mathcal{B}_{\mathrm{p}} \bar{\Omega} A_{+}, \tilde{b}+\tilde{B}\right)=\lim _{\leftarrow}\left(\mathcal{B}_{\mathrm{p}} \bar{\Omega}\left(A_{i}\right)_{+}, \tilde{b}+\tilde{B}\right)
$$

the short sequence

$$
0 \rightarrow \lim _{\overleftarrow{i}}^{1} H P_{n+1}\left(A_{i}\right) \rightarrow H P_{n}(A) \rightarrow \lim _{\overleftarrow{i}} H P_{n}\left(A_{i}\right) \rightarrow 0
$$

is exact for each $n$. The same method works for the homology groups $H_{*}^{\text {naive }}, H_{*}^{\text {bar }}, H H_{*}$ and $H C_{*}$. 


\section{Fréchet algebras as projective limits of Banach algebras}

Let $A$ be a Fréchet locally $m$-convex algebra. We may assume that the sequence of submultiplicative semi-norms $\Gamma=\left(p_{i}\right)_{i \in \mathbb{N}}$ on $A$ is increasing; that is, for every $a \in A$,

$$
p_{1}(a) \leqslant p_{2}(a) \leqslant \cdots p_{n}(a) \leqslant p_{n+1}(a) \leqslant \cdots .
$$

$[33, \S I .3]$

The Arens-Michael decomposition asserts that, up to topological isomorphism of algebras, $A=\lim _{\overleftarrow{i}} A_{i}$, the projective limit of a sequence of Banach algebras $A_{i}$ (see, for example, [33, Corollary III.3.2]). Here $A_{i}$ is the completion of the normed algebra $\left(A / p_{i}^{-1}(0), p_{i}\right)$. We shall denote the closed two-sided ideal

$$
p_{i}^{-1}(0)=\operatorname{Ker} p_{i}=\left\{a \in A \mid p_{i}(a)=\{0\}\right\}
$$

of $A$ by $N_{i}$. For any $n$, the quotient map

$$
\rho_{n}: A \rightarrow A / N_{n}
$$

is a continuous surjective algebra morphism. Consider also the mapping $\bar{\rho}_{n}: A \rightarrow A_{n}$ obtained by composing $\rho_{n}$ with the natural embedding $i_{n}: A / N_{n} \rightarrow A_{n}$. Recall that, in view of [33, §III.3], for any $n$ and $m$, with $n \leqslant m$, the mapping

$$
f_{n m}: A / N_{m} \rightarrow A / N_{n}: f_{n m}\left(a+N_{m}\right) \mapsto a+N_{n}
$$

is a continuous surjective algebra morphism and one has the relation

$$
\rho_{n}=f_{n m} \circ \rho_{m}
$$

for any $n, m$, with $n \leqslant m$. Furthermore, by considering the continuous extensions $\bar{f}_{n m}$ of the maps $f_{n m} \circ i_{n}$, one can see that the family $\left\{\left(A_{n}, \bar{f}_{n m}\right)_{\mathbb{N}}\right\}$ is a reduced countable projective system of Banach algebras. Therefore, the following result follows from Theorems 5.4 and 5.1.

Theorem 6.1. Let $A$ be a Fréchet locally $m$-convex algebra and $A=\lim _{\leftarrow} A_{i}$. Then, for the cohomology groups $H=H_{\text {naive }}, H_{\mathrm{bar}}, H H$ and $H C$, up to isomorphism of linear spaces, for all $n \geqslant 0$,

$$
H^{n}(A)=\lim _{\vec{i}} H^{n}\left(A_{i}\right)
$$

and, for $k=0,1$,

$$
H P^{k}(A)=\lim _{\vec{i}} H P^{k}\left(A_{i}\right)
$$

and, for homology groups $H=H^{\text {naive }}, H^{\text {bar }}, H H, H C, H P$, the short sequence

is exact.

$$
0 \rightarrow \lim _{\overleftarrow{i}}^{1} H_{n+1}\left(A_{i}\right) \rightarrow H_{n}(A) \rightarrow \lim _{\overleftarrow{i}} H_{n}\left(A_{i}\right) \rightarrow 0
$$


Recall that $b_{0}: A \hat{\otimes} A \rightarrow A$ is uniquely determined by $a \otimes b \mapsto a b-b a$.

Theorem 6.2. Let $A$ be a Fréchet locally $m$-convex algebra and $A=\lim _{\overleftarrow{i}} A_{i}$. Suppose that each Banach algebra $A_{i}$ is biflat. Then:

(i) for all $\ell \geqslant 0, H C_{2 \ell}(A)=A /[A, A]$ and $H C_{2 \ell+1}(A)=\{0\}$;

(ii) $H P_{0}(A)=A /[A, A]$ and $H P_{1}(A)=\{0\}$;

(iii) for all $\ell \geqslant 0, H C^{2 \ell}(A)=A^{\text {tr }}$ and $H C^{2 \ell+1}(A)=\{0\}$;

(iv) $H P^{0}(A)=A^{\text {tr }}$ and $H P^{1}(A)=\{0\}$.

Proof. As mentioned in $\S 4.3$, for any biflat Banach algebra $A_{i}$, it is known that $H_{n}^{\text {bar }}\left(A_{i}\right)=\{0\}$ for all $n \geqslant 0$ and hence, by [5, Lemma 2], that $H_{\text {bar }}^{n}\left(A_{i}\right)=\{0\}$ for all $n \geqslant 0$. Therefore, by Theorem $6.1, H_{\text {bar }}^{n}(A)=\{0\}$ for all $n \geqslant 0$, and so, by [5, Lemma 2], $H_{n}^{\text {bar }}(A)=\{0\}$ for all $n \geqslant 0$. By $[\mathbf{5}, \S 3]$, for all $n \geqslant 0, H_{n}^{\text {naive }}(A)=H H_{n}(A)$ and $H_{\text {naive }}^{n}(A)=H H^{n}(A)$.

By $\left[\mathbf{2 1}\right.$, Theorem 25], for any biflat Banach algebra $A_{i}$, it is known that $H_{\text {naive }}^{n}\left(A_{i}\right)=$ $\mathcal{H}^{n}\left(A_{i}, A_{i}^{\prime}\right)=\{0\}$ for all $n \geqslant 1$. Hence, by Theorem 6.1 ,

$$
H H^{n}(A)=H_{\text {naive }}^{n}(A)=\lim _{\vec{i}} H_{\text {naive }}^{n}\left(A_{i}\right)=\{0\} \quad \text { for all } n \geqslant 1 \text {. }
$$

By Proposition 2.4, $H H_{n}(A)=\{0\}$ for all $n \geqslant 1$. Note that, by definition, $H_{0}^{\text {naive }}(A)=$ $A / \operatorname{Im} b_{0}$ and $H_{\text {naive }}^{0}(A)=A^{\text {tr }}$. By Proposition 2.4 , the fact that

$$
H_{\text {naive }}^{n}(A)=\{0\} \quad \text { for all } n \geqslant 1
$$

implies that $H_{0}^{\text {naive }}(A)$ is Hausdorff and therefore that $H_{0}^{\text {naive }}(A)=A /[A, A]$. Statements (i) and (iii) follow from the exactness of the long Connes-Tsygan sequences of continuous homology and cohomology groups; see Theorem 3.1. Statements (ii) and (iv) follow from Propositions 3.2 and 3.3 for $N=1$. In view of [20, Lemma 0.5.9] or [2, Lemma 7.1.32], one can deduce that, for homology groups, these isomorphisms are topological isomorphisms.

Example 6.3. Let $C(\mathbb{R})$ be the Fréchet locally $m$-convex algebra of continuous complex-valued functions on $\mathbb{R}$. Here an Arens-Michael decomposition is $C(\mathbb{R})=$ $\lim _{\overleftarrow{k}} C[-k, k]$. Each $C^{*}$-algebra $C[-k, k]$ is amenable and therefore biflat. By Theorem 6.2 , since $C(\mathbb{R})$ is commutative, for all $\ell \geqslant 0$,

$$
\begin{aligned}
& H P_{0}(C(\mathbb{R}))=H C_{2 \ell}(C(\mathbb{R}))=C(\mathbb{R}), \\
& H P_{1}(C(\mathbb{R}))=H C_{2 \ell+1}(C(\mathbb{R}))=\{0\}, \\
& H P^{0}(C(\mathbb{R}))=H C^{2 \ell}(C(\mathbb{R}))=C(\mathbb{R})^{\prime}=\lim _{\vec{k}} M([-k, k])=M_{\mathrm{c}}(\mathbb{R}),
\end{aligned}
$$

the complex linear space of $\mathbb{C}$-valued Radon measures on $\mathbb{R}$ with compact support, and $H P^{1}(C(\mathbb{R}))=H C^{2 \ell+1}(C(\mathbb{R}))=\{0\}$. 
Corollary 6.4. Let $A$ be a Fréchet locally $m$-convex algebra and $A=\lim _{\leftarrow} A_{i}$. Suppose that, for each Banach algebra $A_{i}, H_{\text {bar }}^{n}\left(A_{i}\right)=H_{\text {naive }}^{n}\left(A_{i}\right)=\{0\}$ for all $n \geqslant 0$. Then, for all $n \geqslant 0, H H^{n}(A)=H H_{n}(A)=\{0\}, H C_{n}(A)=H C^{n}(A)=\{0\}$, and, for $k=0,1$, $H P_{k}(A)=H P^{k}(A)=\{0\}$.

Corollary 6.5. Let $A$ be a Fréchet locally $C^{*}$-algebra and $A=\lim _{\leftarrow} A_{i}$. Suppose that each $C^{*}$-algebra $A_{i}$ has no non-zero bounded trace. Then, for all $n \geqslant 0, H H^{n}(A)=$ $H H_{n}(A)=\{0\}, H C_{n}(A)=H C^{n}(A)=\{0\}$, and, for $k=0,1, H P_{k}(A)=H P^{k}(A)=\{0\}$.

Proof. It is known that for a $C^{*}$-algebra $A_{i}$ without non-zero bounded traces $H_{\text {bar }}^{n}\left(A_{i}\right)=H_{\text {naive }}^{n}\left(A_{i}\right)=\{0\}$ for all $n \geqslant 0$ (see $\left.\S 4.4\right)$. The result follows from Corollary 6.4 .

In the next example we shall consider cyclic homology and cohomology of locally $C^{*}$-algebras $\mathcal{L}(H)$, which play a similar role in the theory of locally $C^{*}$-algebras to that of the $C^{*}$-algebra of all bounded linear operators on a Hilbert space in the theory of $C^{*}$-algebras $[\mathbf{2 2}, \S 5]$.

Example 6.6. Let $H=\lim _{\vec{i}} H_{i}$ be a strict inductive limit of Hilbert spaces, that is,

$$
H=\bigcup_{m=1}^{\infty} H_{m},
$$

where $\left(H_{m},\langle\cdot, \cdot\rangle_{m}\right)_{m=1}^{\infty}$ is an increasing sequence of Hilbert spaces

$$
H_{1} \subset H_{2} \subset \cdots \subset H_{m} \subset H_{m+1} \subset \cdots
$$

such that $\langle\cdot, \cdot\rangle_{i}=\langle\cdot, \cdot\rangle_{j}$ on $H_{i}$ for all $i<j$. We endow $H$ with the $L F$-topology. Suppose that $H_{1}$ and $H_{m+1} / H_{m}, m=1,2, \ldots$, are infinite-dimensional spaces. Consider the Fréchet locally $C^{*}$-algebra $\mathcal{L}(H)$ of continuous linear operators $T$ on $H$ that leave each $H_{i}$ invariant and satisfy $T_{j} P_{i j}=P_{i j} T_{j}$ for all $i<j$, where $T_{j}=T \mid H_{j}: T_{j}(\eta)=T(\eta)$ for $\eta \in H_{j}$ and $P_{i j}$ is the projection from $H_{j}$ onto $H_{i}$. By the Arens-Michael decomposition, up to topological isomorphism of algebras,

$$
\mathcal{L}(H)=\lim _{\overleftarrow{m}} \mathcal{L}\left(H_{m}\right)
$$

the projective limit of a sequence of $C^{*}$-algebras $\mathcal{L}\left(H_{m}\right)$ where, for each $m, \mathcal{L}\left(H_{m}\right)$ is isomorphic to the $C^{*}$-direct sum

$$
\mathcal{B}\left(H_{1}\right) \oplus \mathcal{B}\left(H_{2} \ominus H_{1}\right) \cdots \oplus \mathcal{B}\left(H_{m} \ominus H_{m-1}\right) .
$$

Here $\mathcal{B}(K)$ is the $C^{*}$-algebra of all bounded linear operators on the Hilbert space $K$ and, as we mentioned in Example 4.9, $\mathcal{B}(K)$ has no bounded trace when $K$ is infinite dimensional. Therefore, by Corollary 6.5, for all $n \geqslant 0, H H^{n}(\mathcal{L}(H))=H H_{n}(\mathcal{L}(H))=\{0\}$, $H C_{n}(\mathcal{L}(H))=H C^{n}(\mathcal{L}(H))=\{0\}$ and, for $k=0,1, H P_{k}(\mathcal{L}(H))=H P^{k}(\mathcal{L}(H))=\{0\}$.

Acknowledgements. The author is grateful to the referee for valuable comments and suggestions and for pointing out several gaps in the first version of the paper. 


\section{References}

1. J. H. Anderson, Commutators of compact operators, J. Reine Angew. Math. 291 (1977), 128-132.

2. C. Banica And O. Stanasila, Méthodes algébriques dans la théorie globale des espaces complexes (Gauthier-Villars, Paris, 1977).

3. N. Bourbaki, Éléments de mathématique. Topologie générale, Chapters 1-4 (Hermann, Paris, 1971).

4. N. Bourbaki, Algèbre homologique (Masson, Paris, 1980).

5. J. BRodzki AND Z. A. LyKova, Excision in cyclic type homology of Fréchet algebras, Bull. Lond. Math. Soc. 33 (2001), 283-291.

6. J. Brodzki and R. Plymen, Periodic cyclic homology of certain nuclear algebras, $C$. $R$. Acad. Sci. Paris I 329 (1999), 671-676.

7. H. Cartan and S. Eilenberg, Homological algebra (Princeton University Press, 1956).

8. E. Christensen And A. M. Sinclair, On the vanishing of $\mathcal{H}^{n}\left(A, A^{*}\right)$ for certain $C^{*}$ algebras, Pac. J. Math. 137 (1989), 55-63.

9. A. Connes, Noncommutative geometry (Academic Press, London, 1994).

10. J. Cuntz, Cyclic theory and the bivariant Chern-Connes character, in Noncommutative geometry, Lecture Notes in Mathematics, vol. 1831, pp. 73-135 (Springer, 2004).

11. J. Cuntz And D. Quillen, Operators on noncommutative differential forms and cyclic homology, in Geometry, Topology and Physics, Conf. Proc., Lecture Notes in Geometrical Topology, vol. VI, pp. 77-111 (International Press, Cambridge, MA, 1995).

12. J. Dixmier, Les $C^{*}$-algèbres et leurs représentations (Gauthier-Villars, Paris, 1969).

13. P. G. Dixon, Left approximate identities in algebras of compact operators on Banach spaces, Proc. R. Soc. Edinb. A 104 (1986), 169-175.

14. J. EschmeIER AND M. Putinar, Spectral decompositions and analytic sheaves, London Mathematical Society Monographs, New Series, vol. 10 (Clarendon Press, Oxford, 1996).

15. T. FACK, Finite sums of commutators in $C^{*}$-algebras, Annls Inst. Fourier 32 (1982), 129-137.

16. M. Fragoulopoulou, Structure of contractible locally $C^{*}$-algebras, Proc. Am. Math. Soc. 129 (2001), 2889-2896.

17. N. Gronbaek, B. E. Johnson And G. A. Willis, Amenability of Banach algebras of compact operators, Israel J. Math. 87 (1994), 289-324.

18. U. HAAgERUP, All nuclear $C^{*}$-algebras are amenable. Invent. Math. 74 (1983), 305-319.

19. P. R. Halmos, Commutators of operators, Am. J. Math. 74 (1952), 237-240 and 76 (1954), 191-198.

20. A. YA. Helemskir, The homology of Banach and topological algebras (Moscow University Press, Moscow, 1986) (in Russian) (Engl. transl. Kluwer Academic Publishers, Dordrecht, 1989).

21. A. YA. HelemskiI, Banach cyclic (co)homology and the Connes-Tsygan exact sequence. J. Lond. Math. Soc. 46 (1992), 449-462.

22. A. Inoue, Locally $C^{*}$-algebras, Mem. Fac. Sci. Kyushu Univ. A 25 (1971), 197-235.

23. H. JARCHOW, Locally convex spaces (B. G. Teubner, Stuttgart, 1981).

24. B. E. Johnson, Cohomology of Banach algebras, Mem. Am. Math. Soc. 127 (1972), iii + 96 pp.

25. C. KAssel, Cyclic homology, comodules, and mixed complexes, J. Alg. 107 (1987), 195216.

26. M. KhalkHALI, Algebraic connections, universal bimodules and entire cyclic cohomology, Commun. Math. Phys. 161 (1994), 433-446.

27. G. Кӧтне, Topological vector spaces, vol. I, Die Grundlehren der mathematischen Wissenschaften, vol. 159 (Springer, 1979). 
28. G. KÖTHE, Topological vector spaces, vol. II, Die Grundlehren der mathematischen Wissenschaften, vol. 237 (Springer, 1979).

29. J.-L. LodAY, Cyclic homology (Springer, 1992).

30. Z. A. Lykova, Excision in Banach simplicial and cyclic cohomology, Proc. Edinb. Math. Soc. 41 (1998), 411-427.

31. Z. A. Lykova, Relative cohomology of Banach algebras, J. Operat. Theory 41 (1999), $23-53$.

32. S. MacLane, Homology (Springer and Academic Press, 1963).

33. A. Mallios, Topological algebras: selected topics, Notas de Matematicá, North-Holland Mathematics Studies, no. 124 (North-Holland, Amsterdam, 1986).

34. G. J. Murphy, $C^{*}$-algebras and operator theory (Academic Press, London, 1990).

35. O. S. OGNEvA, Equality of the homological dimension of the Fréchet algebra of smooth functions on a manifold with the dimension of the manifold, Funkt. Analysis i Pril. 20 (1986), 92-93 (in Russian) (Engl. transl. Funct. Analysis Applic. 20 (1986), 248-250).

36. O. S. Ogneva And A. Ya. Helemskit, Homological dimensions of certain algebras of fundamental (test) functions, Mat. Zametki 35 (1984), 177-187 (in Russian) (Engl. Transl. Math. Notes 35 (1984), 93-99).

37. V. P. PAlamodov, Homological methods in the theory of locally convex spaces, Usp. Mat. Nauk 26 (1971), 3-65 (in Russian) (Engl. transl. Russ. Math. Surv. 26 (1971), 1-64).

38. TH. W. PAlmer, Banach algebras and the general theory of *-algebras, vol. I, Algebras and Banach algebras (Cambridge University Press, 1994).

39. A. L. T. PAterson, Virtual diagonals and $n$-amenability for Banach algebras. Pac. J. Math. 175 (1996), 161-185.

40. A. L. T. PAterson And R. R. Smith, Higher-dimensional virtual diagonals and ideal cohomology for triangular algebras, Trans. Am. Math. Soc. 349 (1997), 1919-1943.

41. S. SAKAI, $C^{*}$-algebras and $W^{*}$-algebras (Springer, 1971).

42. Yu. V. Selivanov, Cohomology of biflat Banach algebras with coefficients in dual bimodules, Funkt. Analysis i Pril. 29 (1995), 84-87 (in Russian) (Engl. transl. Funct. Analysis Applic. 29 (1995), 289-291).

43. Yu. V. Selivanov, Cohomological characterizations of biprojective and biflat Banach algebras. Monatsh. Math. 128 (1999), 35-60.

44. J. L. TAYLOR, Homology and cohomology for topological algebras, Adv. Math. 9 (1972), $137-182$.

45. J. L. TAYLOR, A general framework for a multi-operator functional calculus, Adv. Math. 9 (1972), 182-152.

46. A. Wassermann, Cyclic cohomology of algebras of smooth functions on orbifolds, in Operator algebras and applications, vol. I (ed. D. E. Evans and M. Takesaki), London Mathematical Society Lecture Note Series, vol. 135, pp. 229-244 (Cambridge University Press, 1988).

47. C. A. WeIBEL, An introduction to homological algebra, Cambridge Studies in Advanced Mathematics, vol. 38 (Cambridge University Press, 1994).

48. M. Wodzicki, Vanishing of cyclic homology of stable $C^{*}$-algebras, C. R. Acad. Sci. Paris I 307 (1988), 329-334. 